\title{
Generalized Multiphoton Quantum Interference
}

\author{
Max Tillmann, ${ }^{1, *}$ Si-Hui Tan, ${ }^{2}$ Sarah E. Stoeckl, ${ }^{1}$ Barry C. Sanders, ${ }^{3,4}$ Hubert de Guise, ${ }^{5}$ \\ René Heilmann, ${ }^{6}$ Stefan Nolte, ${ }^{6}$ Alexander Szameit, ${ }^{6}$ and Philip Walther ${ }^{1}$ \\ ${ }^{1}$ Faculty of Physics, University of Vienna, Boltzmanngasse 5, A-1090 Vienna, Austria \\ ${ }^{2}$ Singapore University of Technology and Design, 20 Dover Drive, 138682 Singapore \\ ${ }^{3}$ Institute for Quantum Science and Technology, University of Calgary, \\ Calgary, Alberta, T2N 1 N4 Canada \\ ${ }^{4}$ Program in Quantum Information Science, Canadian Institute for Advanced Research, \\ Toronto, Ontario, M5G 128 Canada \\ ${ }^{5}$ Department of Physics, Lakehead University, Thunder Bay, Ontario, P7B 5E1 Canada \\ ${ }^{6}$ Institute of Applied Physics, Abbe Center of Photonics, Friedrich-Schiller Universität Jena, \\ Max-Wien-Platz, 1, D-07743 Jena, Germany
}

(Received 22 April 2015; revised manuscript received 21 July 2015; published 27 October 2015)

\begin{abstract}
Nonclassical interference of photons lies at the heart of optical quantum information processing. Here, we exploit tunable distinguishability to reveal the full spectrum of multiphoton nonclassical interference. We investigate this in theory and experiment by controlling the delay times of three photons injected into an integrated interferometric network. We derive the entire coincidence landscape and identify transition matrix immanants as ideally suited functions to describe the generalized case of input photons with arbitrary distinguishability. We introduce a compact description by utilizing a natural basis that decouples the input state from the interferometric network, thereby providing a useful tool for even larger photon numbers.
\end{abstract}

DOI: 10.1103/PhysRevX.5.041015

\section{INTRODUCTION}

The recent development of quantum photonics technology [1] allows experiments using a growing number of photons and large, complex interferometric networks. Manipulating such large Hilbert spaces requires well-adapted tools in both theory and experiment. Although nonclassical interference is often associated with perfectly indistinguishable photons, this represents only the simplest case of photon states fully symmetric under permutation. Experimentally, partial distinguishability is ubiquitous, because the generation of indistinguishable multiphoton states currently remains a challenge. Moreover, partial distinguishability is of fundamental interest, as highlighted, for instance, by the nonmonotonicity of the quantum-to-classical transition $[2,3]$.

The objective of this paper is to show how controllable delays in multimode coincidence experiments are related to the interference of photons of controllable partial distinguishability. This is done by presenting a novel description for the nonclassical interference of multiple photons of arbitrary distinguishability propagating through arbitrary

*max.tillmann@univie.ac.at

Published by the American Physical Society under the terms of the Creative Commons Attribution 3.0 License. Further distribution of this work must maintain attribution to the author(s) and the published article's title, journal citation, and DOI.
Subject Areas: Optics, Quantum Physics,

Quantum Information

interferometers. We introduce a symmetry-adapted and therefore natural basis that plays the role of normal coordinates for the description of the nonclassical interference of photons. In our framework, a different interferometer just depends on a different set of normal coordinates; the degree of nonclassical interference is determined solely by the properties of the photons. Distinguishability, as the central property, is tunable by treating temporal delay as an explicit parameter, thereby allowing access to the whole spectrum of nonclassical interference. In this perspective, our results differ from Tichy [4] and Shchesnovich [5]; these authors also discuss partial distinguishability but in formalisms where time delays, which are our controllable parameters, are not immediately explicit. The work of Tamma and Laibacher [6] also tackles partial distinguishability in a perspective different from ours.

Here, we treat the nonclassical interference of $n=3$ photons injected into different input ports of a linear-optical quantum network and investigate the probability to detect them as an $n$-fold coincidence for the case that the photons leave the interferometer in different output ports. Our approach can be generalized to a higher number of photons $n$; however, the case for $n=3$ allows an intuitive visualization through a three-dimensional coincidence landscape, where two axes span the distinguishability space for the three photons whereas the third axis quantifies the output probability for a coincident detection event. The features of such 
coincidence landscapes are usually understood as the result of nonclassical interference. We formalize this intuition by associating landscape features with immanants, which are polynomial functions in the elements of the scattering submatrix, having definite symmetries under permutations of rows or columns of this submatrix. The permanent and the determinant are special cases of immanants corresponding to fully symmetric or fully antisymmetric functions under permutations of the rows or columns of the scattering submatrix, respectively. More general immanants have mixed permutation symmetries and are related to partial distinguishability of the input photons as in Ref. [7].

Figure 1 shows how the analysis can break the rates of the landscape in terms of contributions from immanants of the submatrix describing the scattering from the input to the output channels. In Fig. 1(b), the landscape is cut along two characteristic directions where landscape features are to be found: the diagonal and antidiagonal line of pairwise temporal delay. The six points sampled in our experiments, indicated by the pins in Figs. 3(b) and 3(e), are chosen because they are located on such landscape features. Details of the setup and data are provided in Sec. V. Although the results for a finite number of pairs of delays are presented in Fig. 3, it is clear from the experimental setup that any part of the landscape can, in principle, be reached by suitably controlling the time delays.

Our work is motivated in part by the resurgence of interest in interference of perfectly indistinguishable photons within the paradigm of BosonSampling. Within this formalism coincidence rates for such indistinguishable photons are related to the modulus square of the permanent of a submatrix of the scattering matrix. Permanents are known to be hard to compute (in the computational complexity sense), and Aaronson and Arkhipov [8] have shown that the distribution of permanents occurring when $m$ identical photons are made to interfere in an $n$-channel network $(n \gg m)$ is also hard to compute. Some immanants are known [9] to be in the same complexity class as permanents, so partial distinguishability could be applicable to some hypothetical generalized BosonSampling of partially distinguishable photons if the distributions of immanants could also be shown to be hard to simulate with a classical computer.

\section{REVIEW OF THE QUANTUM INTERFERENCE OF TWO BOSONS}

In the seminal experiment by Hong, $\mathrm{Ou}$, and Mandel [10], two photons are injected into distinct input ports of a beam splitter, which is effectively an $m=2$ interferometer, where $m$ is the number of modes of the interferometer. One element of the output probability distribution corresponding to the case where the two photons exit the beam splitter in different output ports is recorded via a coincidence measurement. In the following, we assume the detectors to be ideal (for further details, see Appendix A3(a), Eq. (A24)). In Fig. 2 we show how the coincidence probability $P_{c}$ depends on the transformation matrix $B$, here defined by the splitting ratio of the beam splitter, and the distinguishability of the photons. In the prominent
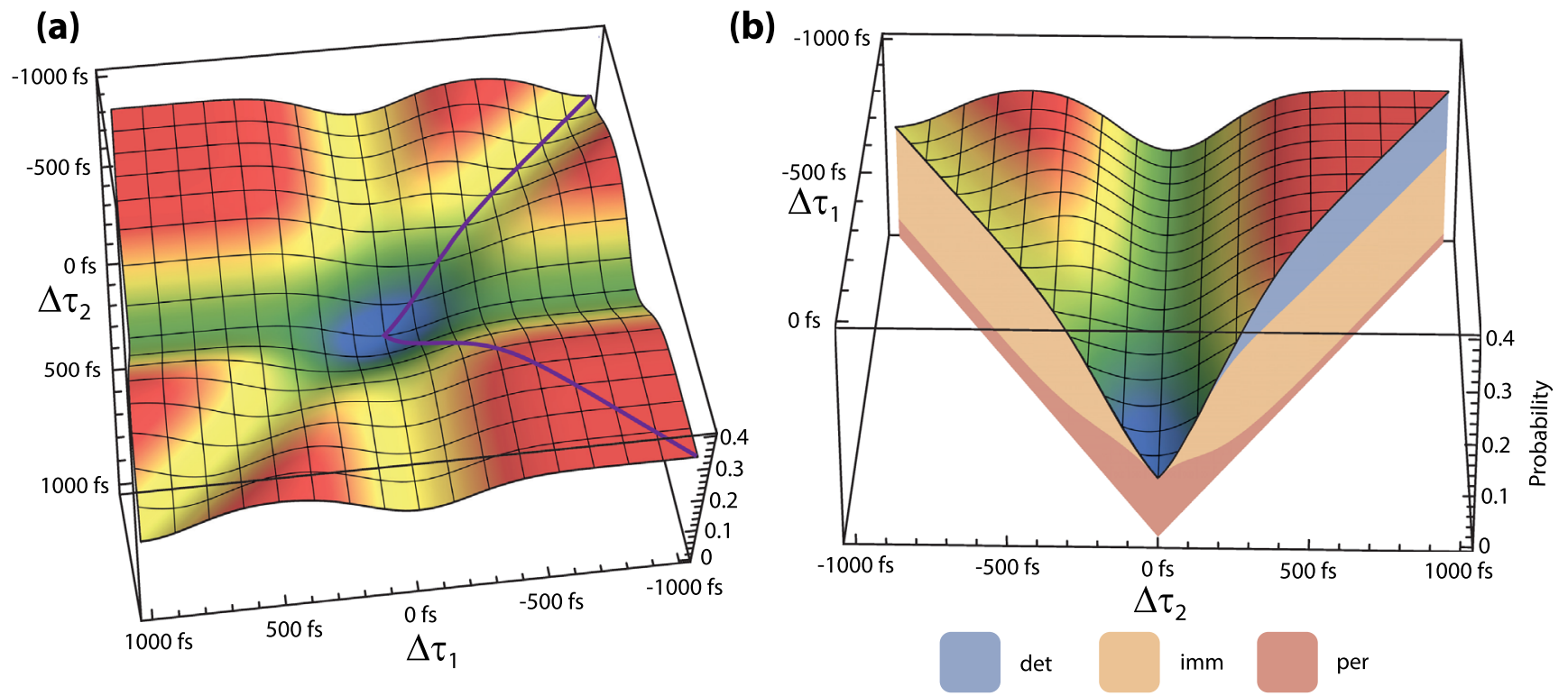

FIG. 1. The coincidence landscape and its substructure. (a) Coincidence landscape which is the visual representation of the nonclassical interference of three photons of tunable distinguishability. (b) Cut along the diagonal and antidiagonal lines of pairwise delays $\Delta \tau_{1}=\Delta \tau_{2}$ and $\Delta \tau_{1}=-\Delta \tau_{2}$, also indicated as a purple trajectory in (a). The height of the landscape corresponds to the coincidence output probability of a three-photon scattering event. The substructure, expressed in terms of contributions from the permanent "per", the determinant "det", and immanants "imm", reveals the nature of the scattering event. Beside the plateaux (color coded in red), which can be explained classically, the whole landscape is governed by a genuine quantum interference of the photons. 


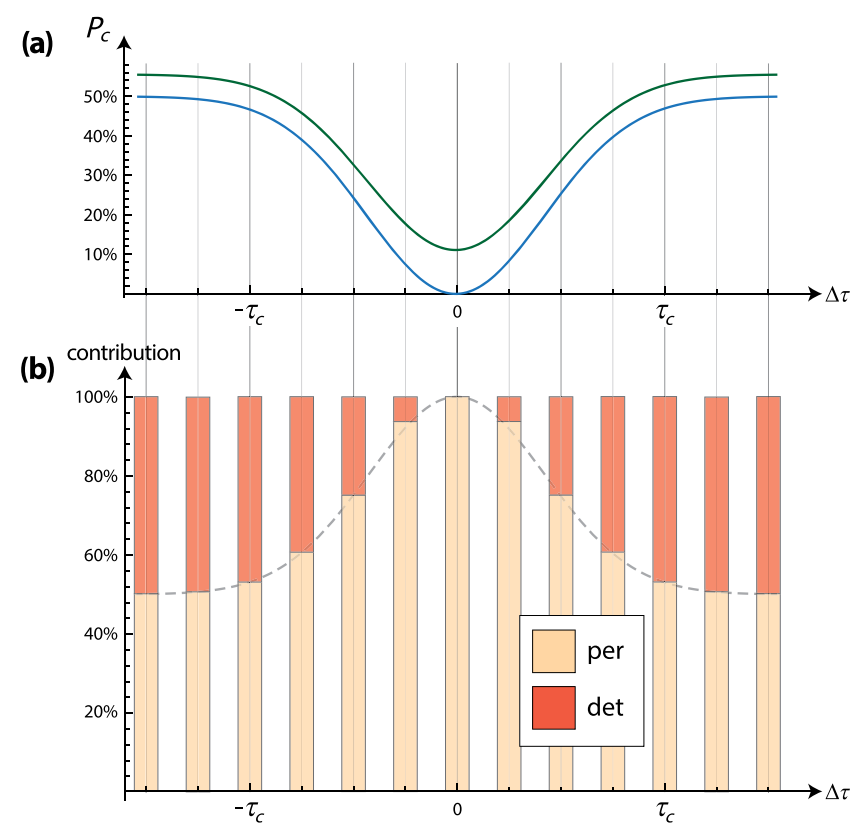

FIG. 2. Two-photon nonclassical interference. Two photons of temporal coherence $\tau_{c}$ enter a beam splitter through different input ports. (a) The coincidence output probability $P_{c}$ that they leave in two different output ports is plotted with respect to a relative temporal delay $\Delta \tau$. This delay is used to tune the distinguishability of the otherwise identical photons. The blue curve shows $P_{c}$ for a 50/50 beam splitter, and the green one for a $67 / 33$ beam splitter. (b) Contribution of the permanent (per) and determinant (det) to the output probability for a coincident detection event $P_{c}$. It is the same for both beam splitters because this description is independent of the interferometer. In the case for zero delay $(\Delta \tau=0)$, only the permanent contributes. By explicitly calculating the permanent, which is zero for a $50 / 50$ beam splitter, the vanishing coincidence probability $P_{c}$ (a) for zero delay is obtained.

example of a balanced, i.e., 50/50, beam splitter and perfectly indistinguishable photons, i.e., also zero temporal delay, the coincidence rate vanishes. The established technique to calibrate for the point of maximal nonclassical interference relies on tuning the relative temporal delay $\Delta \tau$ and therefore the distinguishability between the two photons. This is described by an overlap integral that accounts for the key properties of the photons such as spectral shape, polarization, and spatial mode, in addition to the relative temporal delay.

The results depend on the permutational symmetry of the two interfering particles. We consider a basis vector $v$ accounting for the permutational symmetries as a natural basis vector for quantum interference. The components of $v$ are matrix functions having definite permutation symmetries in the entries of the unitary network matrix $B$. The first component is chosen to be the permanent (per) of the matrix, which is fully symmetric under permutation of rows or columns; the second component is the determinant (det) and is fully antisymmetric under permutation. These are the only two possible symmetries when permuting two objects. By using the basis vector $\boldsymbol{v}$, we obtain an elegant and compact form expression for the coincidence rate $P_{c}(\Delta \tau)$, in which the rate matrix $\hat{R}^{(2)}(\Delta \tau)$ takes a diagonal form, with entries depending only on properties of the input state:

$$
\begin{aligned}
P_{c}(\Delta \tau)= & \int d \omega \int d \omega^{\prime}\left|\left\langle\psi_{i n_{11}}\left|\hat{B}^{\dagger} \hat{a}_{1}^{\dagger}(\omega) \hat{a}_{2}^{\dagger}\left(\omega^{\prime}\right)\right| 0\right\rangle\right|^{2} \\
:= & \boldsymbol{v}^{\dagger}\left[\hat{R}^{(2)}(\Delta \tau)\right] \boldsymbol{v} \\
= & \left(\begin{array}{c}
\operatorname{per}(B) \\
\operatorname{det}(B)
\end{array}\right)^{\dagger}\left[\frac{1}{2}\left(\begin{array}{cc}
1 & 0 \\
0 & 1
\end{array}\right)\right. \\
& \left.+\frac{1}{2}\left|\beta_{12}(\Delta \tau)\right|^{2}\left(\begin{array}{cc}
1 & 0 \\
0 & -1
\end{array}\right)\right]\left(\begin{array}{c}
\operatorname{per}(B) \\
\operatorname{det}(B)
\end{array}\right),
\end{aligned}
$$

where $\left|\beta_{12}(\Delta \tau)\right|^{2}$ is a distinguishability function originating from the spectrotemporal overlap of two photons,

$$
\beta_{i j}=\int d \omega \alpha_{i}(\omega) \alpha_{j}(\omega) e^{i \omega\left(\tau_{i}-\tau_{j}\right)},
$$

and is equal to $\frac{1}{2} \zeta e^{-\xi \Delta \tau^{2}}$ for single photons of Gaussian spectral shape as used in our experiment. Here, $0 \leq \zeta \leq 1$ is derived from the mode-overlap integral above, $\left|\psi_{i n_{11}}\right\rangle=$ $\left.\hat{A}_{1}^{\dagger}\left(\alpha_{1}\right) \hat{A}_{2}^{\dagger}\left(\alpha_{2}\right) e^{i\left(\omega_{1} \tau_{1}+\omega_{2} \tau_{2}\right.}\right)|0\rangle$ is the state impinging on the beam splitter, and $\xi$ is a factor describing the shape of the interference feature (see Appendix A 1 for further details). Note that the distinguishability parameter $\beta_{i j}$ can be generalized to account for mismatch in any degree of freedom of the photons.

The ratio of the two nonzero entries of the rate matrix $\hat{R}_{11}^{(2)}$ and $\hat{R}_{22}^{(2)}$ reveals the nature of the nonclassical interference of two photons of arbitrary coherence. For indistinguishable photons (where $\zeta=1$ ) and zero temporal delay $\Delta \tau, \hat{R}_{22}^{(2)}$ is also zero and the output probability is proportional to the permanent of $B$ only. The permutationally symmetric nature of identical bosonic particles, e.g., photons, is reflected in transition amplitudes determined by a permutationally symmetric function-the permanent.

Temporal delays larger than the coherence time of the photons, $\Delta \tau \gg \tau_{c}$, result in complete loss of coherence as the mode-overlap integral $\left|\beta_{12}(\Delta \tau)\right|^{2}$ of Eq. (1) converges to zero. In this case, often characterized as classical behavior of two photons, $\hat{R}_{11}^{(2)}=\hat{R}_{22}^{(2)}=(1 / n !)=$ $(1 / 2 !)=0.5$. The state is now an equal mixture of symmetric and antisymmetric parts and does not exhibit any of the indistinguishability features associated with quantum interference.

This analysis can be generalized to the quantum interference of two photons in larger interferometric networks: the two input ports and the two output ports of such a network define $2 \times 2$ scattering submatrices $\tilde{B}$, the vector $v$ now contains matrix functions of $\tilde{B}$ and the rate matrix $\hat{R}^{(2)}(\Delta \tau)$ stays identical, independent of $\tilde{B}$. Figure 2(b) highlights how this natural basis cleanly separates effects 
arising from distinguishability of the input state from effects of the interferometric network: whereas the numerical values of the permanent and the determinant depend on the matrix $B$, the ratio of their contributions depends only on partial distinguishability of the photons, irrespective of the details of the interferometer.

\section{QUANTUM INTERFERENCE OF THREE BOSONS}

Consider a scenario where two photons are nearly indistinguishable and the third is delayed significantly. Adding a third photon leads to situations that can no longer be understood by the weighted sum of the permanent and determinant. In order to describe such a behavior, a more general matrix function, the immanant, is required $[7,11]$. The immanant [12] expands the concept of the permanent and determinant to mixed permutation symmetries; the immanant is defined as

$$
\operatorname{imm}^{\{\lambda\}}(M)=\sum_{\sigma} \chi^{\{\lambda\}}(\sigma) \prod_{i} M_{i \sigma(i)},
$$

for $M_{i j}$ matrix elements of $M$, with $\chi^{\{\lambda\}}(\sigma)$ of the element $\sigma$ in the representation $\{\lambda\}$, and $\sigma(i)$ the result of permuting column $i$ to column $j=\sigma(i)$. The permanent, for which every $\chi^{\{n\}}(\sigma)=1$, and the determinant, for which $\chi^{\left\{1^{n}\right\}}(\sigma)=\operatorname{sgn}(\sigma)$, are special cases of the immanant for the fully symmetric representation (conventionally labeled $\{n\}$ ) and alternating representation (conventionally labeled $\left.\left\{1^{n}\right\}\right)$ of $S_{n}$, the permutation group of $n$ objects. The character table of $S_{3}$ is provided in Table I.

For three photons there is, in addition to the permanent and the determinant, one immanant corresponding to the representation $\{2,1\}$ or Young diagram $\boxminus$ of $S_{3}$. For simplicity, we henceforth refer to this immanant as "the immanant" and refer explicitly to the permanent and determinant as needed. The immanant of $M$ is given by
TABLE I. The character table for $S_{3}$.

\begin{tabular}{|c|c|c|c|c|}
\hline $\begin{array}{l}\text { Elements } \\
\text { irrep } \lambda\end{array}$ & $\begin{array}{c}\mathbb{1} \\
\chi^{\lambda}(\mathbb{1})\end{array}$ & $\begin{array}{c}\left\{P_{12}, P_{13}, P_{23}\right\} \\
\chi^{\lambda}\left(P_{a b}\right)\end{array}$ & $\begin{array}{c}\left\{P_{123}, P_{132}\right\} \\
\chi^{\lambda}\left(P_{a b c}\right)\end{array}$ & Dimension \\
\hline \multirow{3}{*}{$\begin{array}{l}\square \\
\square \\
\theta \\
\theta\end{array}$} & 1 & 1 & 1 & 1 \\
\hline & 2 & 0 & -1 & 2 \\
\hline & 1 & -1 & 1 & 1 \\
\hline
\end{tabular}

Unlike the permanent or the determinant, an immanant does not come back to a multiple of itself if we permute, say, columns 1 and 2 . Indeed, one finds, by permuting columns of $M$, a total of four linearly independent immanants. They can be organized in two pairs of linear combinations of immanants so that, upon permutations, the elements of each pair mix among themselves but not across pairs. Although such behavior under permutation may appear unseemly, it is not at all uncommon:

$$
|\psi\rangle=a_{1}^{\dagger}\left(\omega_{1}\right)\left[a_{2}^{\dagger}\left(\omega_{2}\right) a_{3}^{\dagger}\left(\omega_{3}\right)+a_{3}^{\dagger}\left(\omega_{2}\right) a_{2}^{\dagger}\left(\omega_{3}\right)\right]|0\rangle
$$

transforms back to itself upon the permutation $\omega_{2} \leftrightarrow \omega_{3}$, but not under $\omega_{1} \leftrightarrow \omega_{2}$. Such states of mixed permutation symmetries are common in the many-body physics problem.

In the smallest instance of a three-photon quantum interference, the photons are injected into an $m=3$ mode interferometric network and measured as threefold coincidences at the three output ports. The optical transformation implemented by the interferometer can be any $3 \times 3$ linear optical transformation $\hat{T}$, and the distinguishability of the three photons is arbitrarily tunable by setting the relative temporal delays: $\Delta \tau_{1}$ between the first and second photon and $\Delta \tau_{2}$ between the second and third photon. The coincidence rate $P_{111}\left(\Delta \tau_{1}, \Delta \tau_{2}\right)$ is given by

$$
\begin{aligned}
P_{111}\left(\Delta \tau_{1}, \Delta \tau_{2}\right)= & \int d \omega \int d \omega^{\prime} \int d \omega^{\prime \prime}\left|\left\langle\psi_{i n_{111}}\left|\hat{T}^{\dagger} \hat{a}_{1}^{\dagger}(\omega) \hat{a}_{2}^{\dagger}\left(\omega^{\prime}\right) \hat{a}_{3}^{\dagger}\left(\omega^{\prime \prime}\right)\right| 0\right\rangle\right|^{2} \\
= & \left.\boldsymbol{v}_{\mathbf{3}}^{\dagger} \hat{R}^{(3)}\left(\Delta \tau_{1}, \Delta \tau_{2}\right)\right] \boldsymbol{v}_{\mathbf{3}} \\
= & \left(\hat{P} \hat{S} \boldsymbol{v}_{\mathbf{3}}\right)^{\dagger}\left[\mathbb{1}+\rho_{12}\left|\beta_{12}\right|^{2}+\rho_{23}\left|\beta_{23}\right|^{2}+\rho_{13}\left|\beta_{13}\right|^{2}+\rho_{132} \beta_{12}^{*} \beta_{23}^{*} \beta_{13}+\rho_{123} \beta_{12} \beta_{23} \beta_{13}^{*}\right]\left(\hat{P} \hat{S} v_{\mathbf{3}}\right) \\
= & \left(\hat{P} \hat{S} \boldsymbol{v}_{\mathbf{3}}\right)^{\dagger}\left[\mathbb{1}+\rho_{12} \zeta_{12} e^{-\xi_{12} \Delta \tau_{1}^{2}}+\rho_{23} \zeta_{23} e^{-\xi_{23} \Delta \tau_{2}^{2}}+\rho_{13} \zeta_{13} e^{-\xi_{13}\left(\Delta \tau_{1}-\Delta \tau_{2}\right)^{2}}\right. \\
& \left.+\zeta_{123}\left(\rho_{132} e^{\xi_{123}^{*}\left(\Delta \tau_{1}, \Delta \tau_{2}\right)}+\rho_{123} e^{\xi_{123}\left(\Delta \tau_{1}, \Delta \tau_{2}\right)}\right)\right]\left(\hat{P} \hat{S} \boldsymbol{v}_{\mathbf{3}}\right),
\end{aligned}
$$

where $\boldsymbol{v}_{\mathbf{3}}, \hat{P}$, and $\hat{S}$ are given explicitly in Eq. (A19). The terms $\beta_{12}, \beta_{23}$, and $\beta_{13}$ are distinguishability parameters defined analogously to Eq. (2). In Eq. (9) these parameters are computed for single photons of Gaussian spectral shape as used in our experiment. Our key point is that, in Eq. (7), the rate matrix $\hat{R}^{(3)}\left(\Delta \tau_{1}, \Delta \tau_{2}\right)$ can always be brought to block-diagonal form, containing two $1 \times 1$ blocks and one $4 \times 4$ block. Indeed, the matrices in Eq. (9) can always be further refined so as to take a final block-diagonal form: 


$$
\begin{aligned}
& \mathbb{1}=\left(\begin{array}{cccccc}
1 & 0 & 0 & 0 & 0 & 0 \\
0 & 1 & 0 & 0 & 0 & 0 \\
0 & 0 & 1 & 0 & 0 & 0 \\
0 & 0 & 0 & 1 & 0 & 0 \\
0 & 0 & 0 & 0 & 1 & 0 \\
0 & 0 & 0 & 0 & 0 & 1
\end{array}\right), \quad \rho_{12}=\left(\begin{array}{cccccc}
1 & 0 & 0 & 0 & 0 & 0 \\
0 & -1 & 0 & 0 & 0 & 0 \\
0 & 0 & 1 & 0 & 0 & 0 \\
0 & 0 & 0 & -1 & 0 & 0 \\
0 & 0 & 0 & 0 & 1 & 0 \\
0 & 0 & 0 & 0 & 0 & -1
\end{array}\right), \\
& \rho_{23}=\left(\begin{array}{cccccc}
1 & 0 & 0 & 0 & 0 & 0 \\
0 & -1 & 0 & 0 & 0 & 0 \\
0 & 0 & -\frac{1}{2} & -\frac{\sqrt{3}}{2} & 0 & 0 \\
0 & 0 & -\frac{\sqrt{3}}{2} & \frac{1}{2} & 0 & 0 \\
0 & 0 & 0 & 0 & -\frac{1}{2} & -\frac{\sqrt{3}}{2} \\
0 & 0 & 0 & 0 & -\frac{\sqrt{3}}{2} & \frac{1}{2}
\end{array}\right), \quad \rho_{13}=\left(\begin{array}{cccccc}
1 & 0 & 0 & 0 & 0 & 0 \\
0 & -1 & 0 & 0 & 0 & 0 \\
0 & 0 & -\frac{1}{2} & \frac{\sqrt{3}}{2} & 0 & 0 \\
0 & 0 & \frac{\sqrt{3}}{2} & \frac{1}{2} & 0 & 0 \\
0 & 0 & 0 & 0 & -\frac{1}{2} & \frac{\sqrt{3}}{2} \\
0 & 0 & 0 & 0 & \frac{\sqrt{3}}{2} & \frac{1}{2}
\end{array}\right) \text {, } \\
& \rho_{123}=\left(\begin{array}{cccccc}
1 & 0 & 0 & 0 & 0 & 0 \\
0 & 1 & 0 & 0 & 0 & 0 \\
0 & 0 & -\frac{1}{2} & -\frac{\sqrt{3}}{2} & 0 & 0 \\
0 & 0 & \frac{\sqrt{3}}{2} & -\frac{1}{2} & 0 & 0 \\
0 & 0 & 0 & 0 & -\frac{1}{2} & -\frac{\sqrt{3}}{2} \\
0 & 0 & 0 & 0 & \frac{\sqrt{3}}{2} & -\frac{1}{2}
\end{array}\right), \quad \rho_{132}=\left(\begin{array}{cccccc}
1 & 0 & 0 & 0 & 0 & 0 \\
0 & 1 & 0 & 0 & 0 & 0 \\
0 & 0 & -\frac{1}{2} & \frac{\sqrt{3}}{2} & 0 & 0 \\
0 & 0 & -\frac{\sqrt{3}}{2} & -\frac{1}{2} & 0 & 0 \\
0 & 0 & 0 & 0 & -\frac{1}{2} & \frac{\sqrt{3}}{2} \\
0 & 0 & 0 & 0 & -\frac{\sqrt{3}}{2} & -\frac{1}{2}
\end{array}\right) \text {. }
\end{aligned}
$$

The vector $\hat{P} \hat{S} v_{\mathbf{3}}$ of Eq. (9) is given explicitly by

$$
\hat{P} \hat{S} v_{3}=\left(\begin{array}{c}
\operatorname{per}(T) \\
\operatorname{det}(T) \\
\frac{1}{2 \sqrt{3}} \operatorname{imm}(T)+\frac{1}{2 \sqrt{3}} \operatorname{imm}\left(T_{312}\right) \\
\frac{1}{6} \operatorname{imm}(T)-\frac{1}{3} \operatorname{imm}\left(T_{132}\right)-\frac{1}{6} \operatorname{imm}\left(T_{213}\right)+\frac{1}{3} \operatorname{imm}\left(T_{312}\right) \\
\frac{1}{6} \operatorname{imm}(T)+\frac{1}{3} \operatorname{imm}\left(T_{132}\right)+\frac{1}{6} \operatorname{imm}\left(T_{213}\right)+\frac{1}{3} \operatorname{imm}\left(T_{312}\right) \\
-\frac{1}{2 \sqrt{3}} \operatorname{imm}(T)+\frac{1}{2 \sqrt{3}} \operatorname{imm}\left(T_{213}\right), .
\end{array}\right)
$$

where $T_{i j k}$ is the matrix $T$ in which rows 1,2 , and 3 have been rearranged in order $i, j, k$. In addition, $\hat{a}_{1}^{\dagger}(\omega), \hat{a}_{2}^{\dagger}\left(\omega^{\prime}\right)$, and $\hat{a}_{3}^{\dagger}\left(\omega^{\prime \prime}\right)$ are the creation operators in modes $1,2,3$ of $T$ for photons with different spectral shape functions dependent on the frequency variables $\omega, \omega^{\prime}, \omega^{\prime \prime}$. Here, $\left|\psi_{i n_{111}}\right\rangle=$ $\hat{A}_{1}^{\dagger}\left(\omega_{1}\right) \hat{A}_{2}^{\dagger}\left(\omega_{2}\right) \hat{A}_{3}^{\dagger}\left(\omega_{3}\right) e^{i\left(\omega_{1} \tau_{1}+\omega_{2} \tau_{2}+\omega_{3} \tau_{3}\right)}|0\rangle$ is the threephoton state impinging on the interferometer, with $A_{k}^{\dagger}\left(\omega_{k}\right)$ defined in Eq. (A2).

The form of the matrices in Eq. (10) is fully dictated by the theory of the group of permutation of three objects: the blocks in Eq. (10) correspond to irreducible representations of this group. The block diagonalization can be done using several methods; one algorithm can be found in Chap. 4 of Ref. [13]. No further reduction into smaller blocks is possible; i.e., the vector $\hat{P} \hat{S} \boldsymbol{v}_{\mathbf{3}}$ is optimal, in the sense that it is the vector (up to a choice of which matrices $\rho$ are diagonalized) that will produce the simplest form of the rates. Indeed, an expression for the coincidence rate of Eq. (6), expanded in terms of immanants, determinants, and permanents, is also given in Eq. (A12); it is a linear superposition of 60 terms. However, utilizing a symmetry-adapted basis allows for the compact expression given in Eqs. (9) and (7) (see Appendix A 2 for further details). Here, the four linearly independent immanants, the permanent, and the determinant of $T$ constitute the components of a six-dimensional basis vector $\hat{P} \hat{S} \boldsymbol{v}_{\mathbf{3}}$.

Much like Eq. (1), the various $\zeta$ terms of Eq. (9) are derived from the mode-overlap integral while the $\xi$ terms describe the shape of the interference feature. In this 
notation the overlap terms weight a sum of six matrices: the identity matrix and five permutation matrices $\rho_{12}, \rho_{13}, \rho_{23}$, $\rho_{123}$, and $\rho_{132}$, the subscripts of which label the permutation operation.

\section{NATURAL BASIS FOR THREE PHOTONS}

Components of the natural basis vector $\hat{P} \hat{S} v_{\mathbf{3}}$ introduced to yield the fully block-diagonal form of Eq. (9) have specific permutation properties: components of one symmetry type transform to components of the same type under permutation; i.e., they are decoupled under permutation. Components of $\hat{P} \hat{S} \boldsymbol{v}_{\mathbf{3}}$ thus play the role of normal coordinates for the nonclassical interference of photons.

Equation (9) highlights the six different permutational possibilities for three photons. Summing the matrices inside the square brackets yields the $6 \times 6$ rate matrix $\hat{R}^{(3)}\left(\Delta \tau_{1}, \Delta \tau_{2}\right)$ of Eq. (7). This $\hat{R}^{(3)}\left(\Delta \tau_{1}, \Delta \tau_{2}\right)$ contains all the information regarding the input state, e.g., mode mismatch and temporal delay, to specify the nonclassical interference of three photons independent of the scattering transformation $T$.

Two entries of the block-diagonal rate matrix are sufficient for an interpretation. $F_{\text {per }}=\left|\hat{R}_{11}^{(3)}\left(\Delta \tau_{1}, \Delta \tau_{2}\right)\right|^{2}$ quantifies the fraction of the output probability distribution proportional to the modulus square of the permanent; the corresponding basis component is fully symmetric under permutation. $F_{\text {det }}=\left|\hat{R}_{66}^{(3)}\left(\Delta \tau_{1}, \Delta \tau_{2}\right)\right|^{2}$ quantifies the fraction of the output probability distribution proportional to the modulus square of the determinant; the corresponding component is antisymmetric under permutation. The contribution proportional to moduli square of the immanants can also be explicitly calculated. When only interested in their overall contribution, it is given as $F_{\text {imm }}=1-F_{\text {per }}-F_{\text {det }}$. In the case of perfectly overlapping photons, $F_{\text {per }}=1$, and therefore only the permanent of the scattering matrix contributes to the output probability distribution. Classical behavior of the photons can be identified for $F_{\text {per }}=F_{\text {det }}=\frac{1}{6}$.

As in the two-photon case, the input state and the interferometer decouple in the natural basis. As a consequence, the treatment of the quantum interference of three photons in larger interferometric networks consisting of many modes becomes very efficient. For such a problem it is sufficient to calculate the rate matrix $\hat{R}^{(3)}$ only once. The scattering matrix $T$, necessary to calculate the basis vector $\boldsymbol{v}_{\mathbf{3}}$ for a specific element of an output probability distribution, is just a $3 \times 3$ submatrix of the larger scattering matrix. It is specified by the input ports of the photons and the ports in which they exit the interferometer. To obtain multiple elements of a probability distribution, it is sufficient to determine their respective components in $\boldsymbol{v}_{\mathbf{3}}$.

\section{COINCIDENCE LANDSCAPE}

In the experiment, four-photon events generated by higher-order emission from a spontaneous parametric down-converter are distributed to four different spatial modes. Relying on a detection event in the trigger mode and postselection, the three-photon input state, one photon in each input mode coupled to the interferometer, is heralded. We ensure that all photons are indistinguishable in a polarization basis. The spectral properties of these photons are independently measured using a single-photon spectrometer. Their relative temporal delay $\Delta \tau_{1}$ and $\Delta \tau_{2}$ can be set using motorized delay lines. The transformation of the femtosecond-written integrated interferometer, a $5 \times 5$ unitary matrix, is recovered using the reconstruction method specified in Appendix A 3.

Injecting the photons in three input ports of the interferometer and detecting them in three separate output ports uniquely selects a $3 \times 3$ scattering submatrix $T$ [see Figs. 3(a) and 3(d)]. For each $3 \times 3$ submatrix, the use of a precisely tunable delay allows us to reveal the full spectrum and thereby the nature of the nonclassical interference. We visualize this as a three-dimensional coincidence landscape as shown in Figs. 3(b) and 3(e). The relief of such a landscape features distinct "landforms" which are in correspondence with distinguishability features of the photons.

In the center region, $\Delta \tau_{1} \approx \Delta \tau_{2} \approx 0 \pm \tau_{c}$, a peak or dip arises due to constructive or destructive interference of all three photons. In the absence of any spectral distinguishability, the absolute zero position $\Delta \tau_{1}=\Delta \tau_{2}=0$ corresponds to $100 \%$ contribution from the permanent of $T$.

Along the three axes $\Delta \tau_{1}=0, \Delta \tau_{2}=0$, and $\Delta \tau_{1}=\Delta \tau_{2}$, valleys or ridges form due to the nonclassical interference of two indistinguishable photons with the third one being partially distinguishable. Along those ridges and valleys the contributions to the output probability come from the permanent and the immanants of the scattering matrix.

"Classical" behavior, i.e., complete distinguishability, of the three photons is associated with plateaus for temporal delays, $\Delta \tau_{1} \approx-\Delta \tau_{2} \gg\left| \pm \tau_{c}\right|$. For such large temporal delays along the antidiagonal axis, all mode-overlap integrals of Eq. (9) converge to zero. Consequently, these are the areas where determinants of the scattering matrix contribute, accounting for the antisymmetrical part of the input state. Coincidences for six points of pairwise different temporal delays, $P 1-P 6$, for two different scattering submatrices [see, for instance, Figs. 3(c) and 3(f)] are measured. These six points are selected because they highlight the connection between landscape features, permutation symmetries, and partial distinguishability. Furthermore, they provide a sufficient set of experimental data for fitting the coincidence landscapes. A reduced $\chi^{2}$ of 1.38 and 1.10 for the two landscapes quantifies the overlap between our theory and the experiment. Our theory assumes a single photon per input mode; hence, the deviations are most 


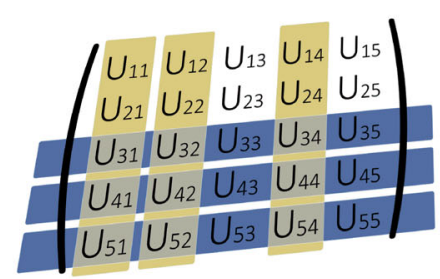

(a)

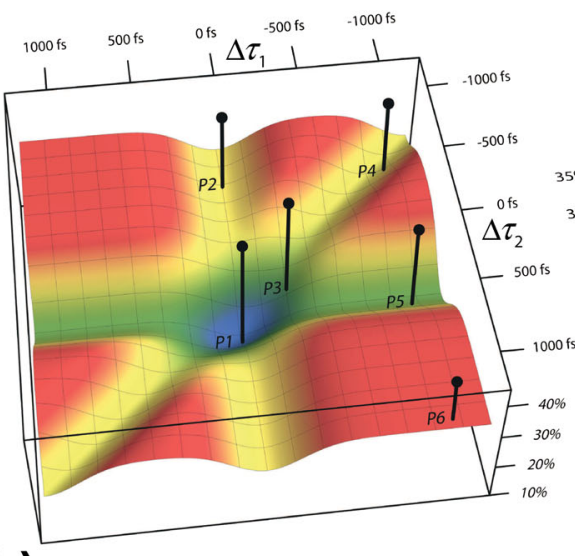

(b)

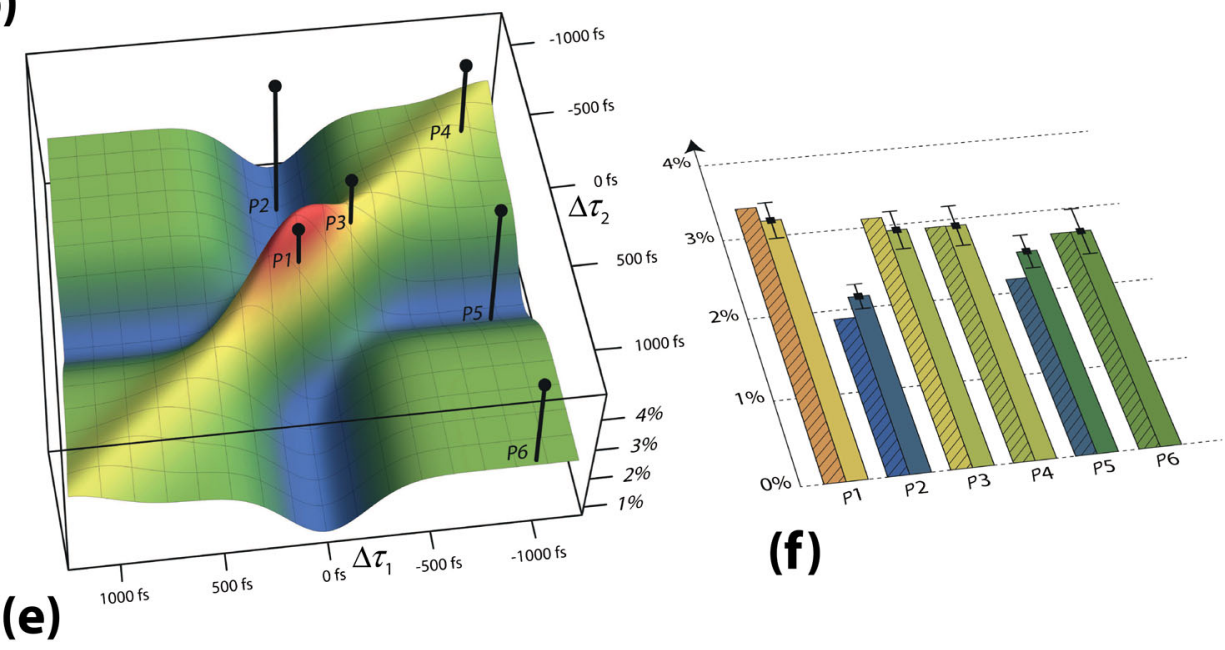

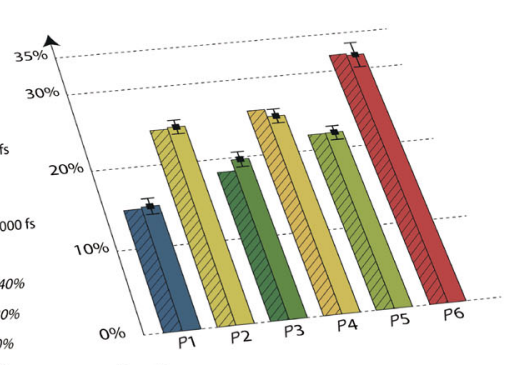

(c) (d)

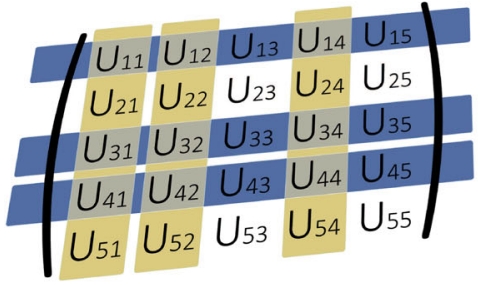

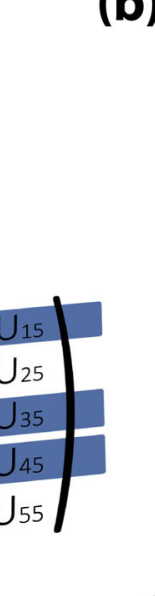

$(e)$

FIG. 3. Three-photon coincidence landscapes. Three photons enter the interferometric network, one each in modes 1 , 2 , and 4 (highlighted in yellow), and exit the network in modes (a) 3, 4, and 5 and (d) 1, 3, and 4, respectively (highlighted in blue). The intersections of the inputs' columns and the outputs' rows uniquely select matrix entries that constitute $3 \times 3$ submatrices. Tuning the temporal delay of the three photons with respect to each other $\left(\Delta \tau_{1}\right.$ and $\left.\Delta \tau_{2}\right)$ gives rise to coincidence landscapes [(b) and (e)]. Their temporal distinguishability determines the degree of nonclassical interference and therefore the probability to detect such an event. Six characteristic points $(P 1-P 6)$ of each landscape are experimentally sampled. Theoretical prediction (left bars, shaded) and experimentally obtained output probabilities (right bars) for the six points and both output combinations are shown in (c) and (f). The reduced $\chi^{2}$ is 1.38 and 1.10 , respectively, and the experimental errors are calculated as standard deviations.

likely due to higher-order emissions and frequency correlations of the input state.

The landscape interpretation can be extended as needed to the interference of larger numbers of photons $n$, which generate $n$-dimensional "hyperlandscapes." These are spanned by $n-1$ axes of pairwise temporal delays with the last axis representing the actual coincidence rate. The landforms range from complex $n$-dimensional features corresponding to the partial indistinguishability of all $n$ photons to the flat plateaus associated with completely distinguishable photons.

\section{A. Systematic exploration of partial distinguishability}

We investigate generalized nonclassical interference of three photons in a five-moded interferometric network in theory and experiment. This serves to illustrate the full permutational spectrum of a generalized nonclassical interference for complex networks exhibiting a generic structure. The photons exhibit slight spectral mismatch and are thus never fully indistinguishable; additionally, the level of partial distinguishability can be increased by controlling temporal delays. Figure 4(a) illustrates the result for a very small degree of partial distinguishability, whereas in Figs. 4(b) and 4(c) the partial distinguishability is increased by varying the temporal delay along a diagonal delay axis $\Delta \tau_{1} \approx \Delta \tau_{2}$. The extreme case of complete distinguishability $\Delta \tau_{1} \approx-\Delta \tau_{2} \gg \tau_{c}$, and thus classical behavior is shown in Fig. 4(d). As a reference we include in all figures the ideal case of zero delay and perfect indistinguishability as gray bars. The group theoretical interferometer-independent contributions $F_{\text {per }}$, $F_{\text {det }}$, and $F_{\text {imm }}$ are contained as an inset in the legend of each figure. 
(a)

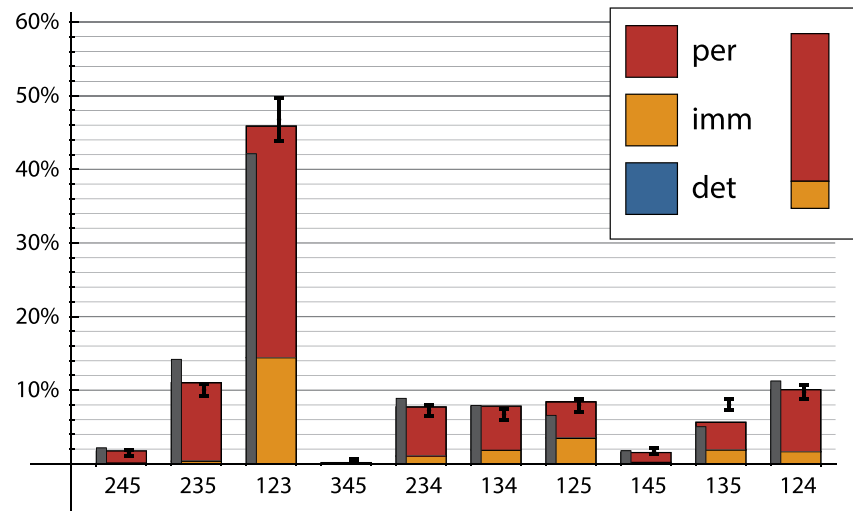

(c)

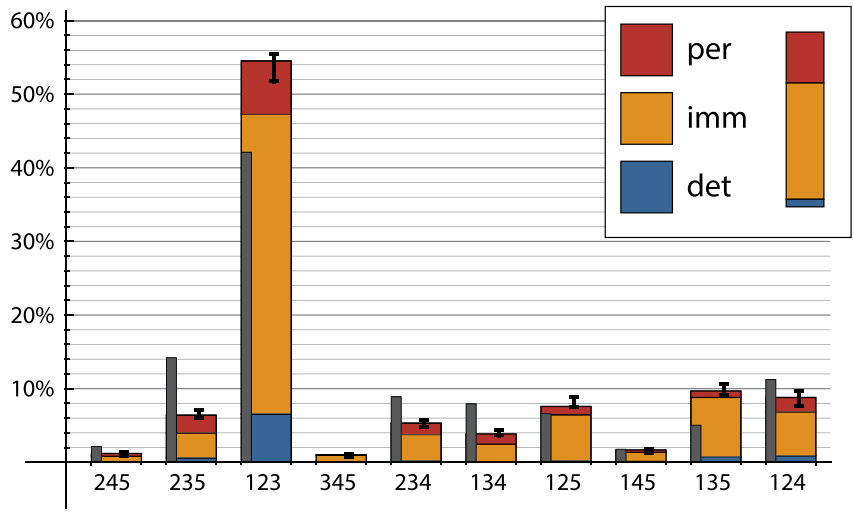

(b)

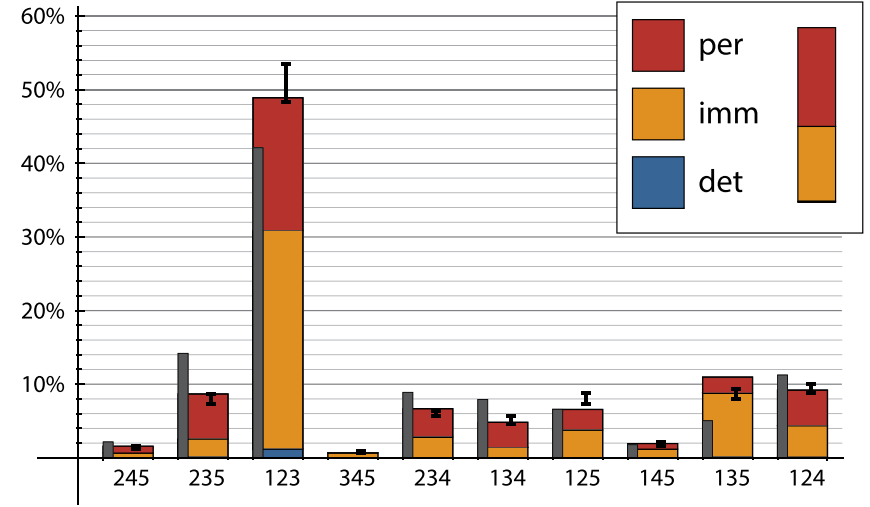

(d)

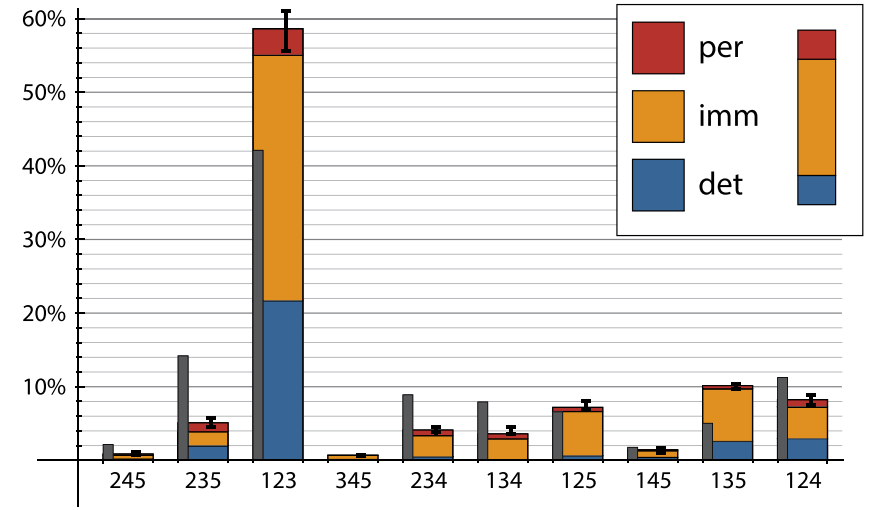

FIG. 4. Experimental output distribution for indistinguishable, semidistinguishable, and distinguishable photons. Various temporal delays for three photons lead to different contributions of permanents, immanants, and determinants. The normalized output probability distributions for the three photons is measured as coincidences from different spatial modes, resulting in ten elements. For all temporal delays the three photons exhibit slight spectral mismatch. Panel (a) depicts the case for a small temporal offset $\Delta \tau_{1} \ll \tau_{c} \gg \Delta \tau_{2}(P 1$ of Fig. 3), whereas for (b) and (c) this delay is increased along a diagonal axis $\Delta \tau_{1} \approx \Delta \tau_{2}$ ( $P 3$ and $P 4$ of Fig. 3). The extreme case of complete distinguishability and therefore classical behavior is shown in (d) ( $P 6$ of Fig. 3). As a reference, the gray bars illustrate the case for perfect indistinguishability and therefore only contribution from the permanent of the scattering submatrix. The interferometer independent contribution $F_{\text {per }}, F_{\text {det }}$, and $F_{\text {imm }}$ is shown in the figure legend. The error bars of the experimental data are standard deviations over 19 independent runs.

The elements of each output probability distribution are recovered by calculating the corresponding matrix functions. Note that for each element the absolute value of these matrix functions, e.g., $|\operatorname{per}(T)|^{2}$ or $|\operatorname{det}(T)|^{2}$, can vary largely depending on the scattering submatrix $T$. This is pronounced for the output event 123 , where $\left|\operatorname{per}\left(T_{123}\right)\right|^{2} \approx \frac{1}{5}\left|\operatorname{det}\left(T_{123}\right)\right|^{2}$. In general, the fraction of the output probability distribution proportional to the permanent drops rapidly with increasing distinguishability. Instead, contributions from immanants become dominant and reflect cases where two of the three photons interfere nonclassically.

For large delays along the diagonal axis $\Delta \tau_{1} \approx \Delta \tau_{2} \gg \tau_{c}$, two photons stay nearly indistinguishable and the contribution from the determinant is suppressed to $F_{\text {det }} \approx 0$ [see Fig. 4(c)]. For comparably large delays along the antidiagonal axis, $\Delta \tau_{1} \approx-\Delta \tau_{2} \gg \tau_{c}$, the three photons' wave functions do not overlap anymore and the determinant contributes with $F_{\text {det }}=\frac{1}{6}$ [see Fig. 4(d)]. This is the case of classical behavior of the three photons [see Fig. 4(d)] and can always be identified by an equal contribution from the permanent and determinant: $F_{\text {per }}=F_{\text {det }}=(1 / 3 !)$ in the case of a three-photon interference and $F_{\text {per }}=F_{\text {det }}=(1 / n !)$ for the nonclassical interference of $n$ photons.

Our theory emphasizes the permutation symmetries of $n$ photons using the representation theory of the symmetric group $S_{n}$. The theory is thus independent of the number of modes $m$ in the interferometer, a feature that is extremely convenient for large-scale networks where $m \gg n$, even if the number of permutations of the output increases as $n$ !.

\section{B. From permanents to immanants}

Quantum computing leverages quantum resources to efficiently perform certain classically hard computations [14]. Whereas many quantum algorithms solve a certain 
decision problem, BosonSampling introduces a new paradigm: it seeks efficient sampling of a distribution of permanents of matrix transformations, which is a task that is hard to implement efficiently on classical computers.

In order to scale BosonSampling to larger instances, two main issues need to be addressed. The first issue is the technology [15-17] needed to increase the size of the instances implemented. The second issue is the handling of possible errors [18-20]. BosonSampling is a purely passive optical scheme and therefore lacks error-correction capabilities [21]. Only in the ideal case where the interfering photons are indistinguishable in all degrees of freedom is the resulting output probability distribution proportional to the permanent only. Our analysis exposes that this condition is rather fragile and therefore distinguishability must be regarded as the dominant source of error. Remarkably, large classes of immanants are known to be in the same complexity class as permanents [22,23]. Thus, it is natural to ask if the output probability distributions depending largely on immanants rather than just the permanent are also computationally hard. Whether this holds for sampling from these distributions is an active field of research.

\section{Generalization to higher number of photons}

The theory presented here in detail for the case of $n=3$ photons can be generalized to a larger number of photons $n$. The rate matrix becomes a matrix of dimension $n ! \times n !$ and carries the so-called regular representation of $S_{n}$. It can be block diagonalized exploiting the same methods used to block diagonalize the regular representation. The algorithm to obtain this block-diagonal form for the rate matrix is described in Table 2 for any $n$-photon input. As highlighted in Sec. VA, the rate matrix is independent of the number of modes in the interferometer. We demonstrate the use of this algorithm for a five-photon input into a nine-mode interferometer. In this specific case of $n=5$, the rate matrix is of dimension $5 !=120$ and can be expressed as $\sum_{\sigma \in S_{5}} \rho_{\sigma} O_{\sigma}$, where $\rho_{\sigma}$ spans a $120 \times 120$ representation $\Gamma$ of $S_{5}$, and where $O_{\sigma}$ contains various delay-dependent factors. The representation $\Gamma$ is reducible and decomposes as

$$
\Gamma=\square \square \oplus 4 \square \square \oplus 5 \square \oplus 6 \boxminus \oplus \oplus \boxminus \oplus 4 \boxminus \oplus \boxminus .
$$

The terms in Eq. (12) are respectively associated with photons of different partial distinguishability: The first term is associated with fully indistinguishable photons, the second term with four photons arriving with no delay and a last photon fully distinguishable with respect to the quartet, and the third term with three photons arriving with no delay and a second pair of photons with no relative delay between themselves. The pair and the trio are fully separable in the temporal domain. The remaining terms can be interpreted in the same fashion, with the last term covering the case of all five photons being fully distinguishable with respect to each other. Each term is labeled by the Young diagram of its partition and associated with an immanant. In general, the coincidence rate is a sum of contributions from modulus squared of linear combinations of these immanants, which can be constructed using the character table for $S_{5}$, as can be found in for instance in Ref. [12]. These linear combinations

TABLE II. Algorithm to block diagonalize the rate matrix.

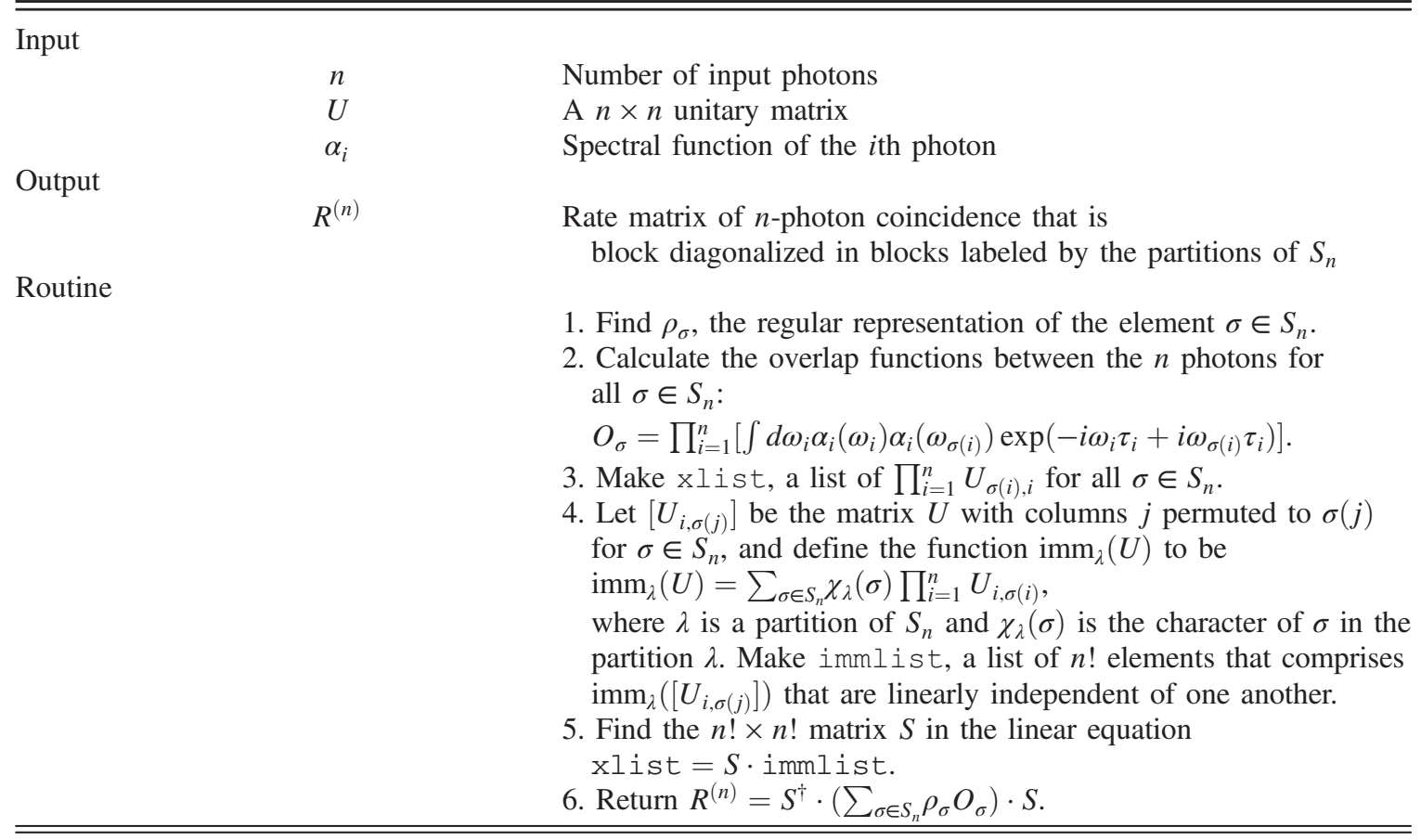


of immanants can be found using, for instance, class operator methods [13].

The algorithm described in Table II can be used to compute the rate matrix that is block diagonalized in the partitions listed in Eq. (12) for five photons propagating through an interferometer. Note that the specific input chosen and the size of the interferometer, in Fig. 5 a $9 \times 9$ interferometer, affect only the basis vectors. For this exemplary case we look at the $\left(\begin{array}{l}9 \\ 5\end{array}\right)=126$ different ways
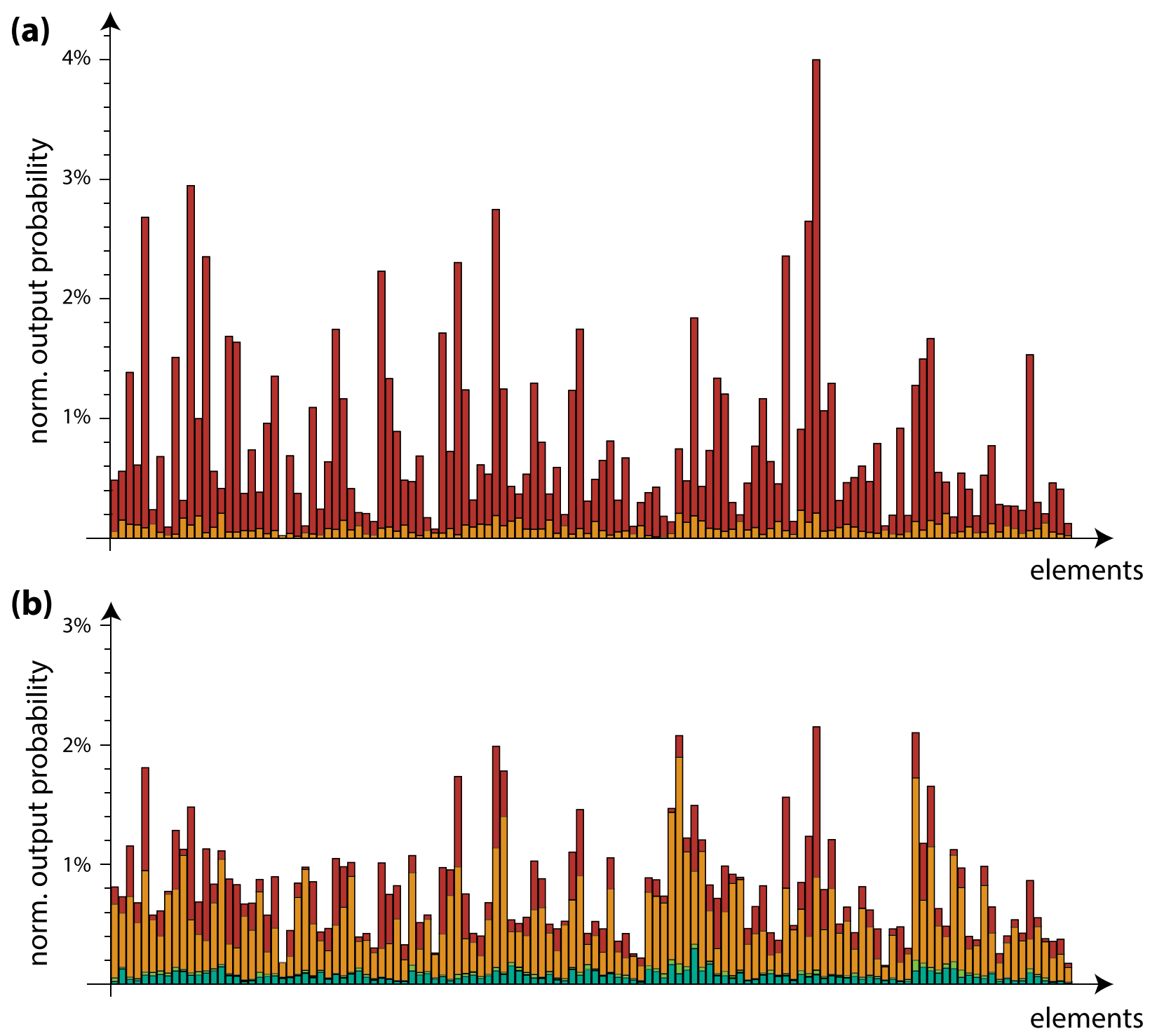

FIG. 5. Five-photon BosonSampling including distinguishability. Simulation of a BosonSampling instance of five photons propagating through an interferometric network of nine modes. The output probability distribution of five photons exiting the interferometer in five different modes is normalized and contains 126 elements. Owing to the large size of this probability distribution, we choose not to list them individually, and we label the horizontal axes by "elements." Panel (a) depicts the close-to-the-ideal case where realistic errors such as slight spectral mismatch and temporal delay $\left(\Delta \tau_{i} \leq \frac{1}{20} \tau_{c}\right)$ of the photons lead to a small degree of partial distinguishability. Panel (b) shows a case where the interfering photons exhibit increased partial distinguishability $\left(\Delta \tau_{i} \leq \frac{1}{5} \tau_{c}\right)$. In these

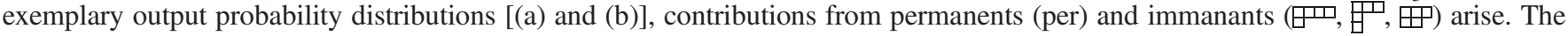
immanant contributions cover physical scenarios with different symmetries under exchange of five photons. The labels of the immanants describe the number of photons that are distinguishable. $\square$, for instance, is the contribution from the case when four photons are indistinguishable from one another but distinguishable from the fifth photon. 
in which five photons can exit a nine-mode interferometer, each photon in a separate mode. Permanents, $\square \square \square$, and immanants of the partitions, $\square \square, \square$, and $\square$, contribute to this 126-element probability distribution and are computed as the trace of their respective block matrices given by the algorithm of Table II. In Fig. 5, this output probability distribution and its substructure is shown for two different degrees of partial distinguishability.

\section{DISCUSSION}

We present a novel analysis of multiphoton quantum interference revealing the full permutational spectrum of input states with arbitrary distinguishability. A comprehensive physical interpretation is achieved by establishing a correspondence between matrix immanants and these mixed symmetry input states. We introduce a rate matrix containing all the information on the nonclassical interference and basis vectors containing the information on the interferometric network. Output probabilities are recovered as an inner product of these vectors with the rate matrix serving as a metric. The rate matrix is block diagonalized and each block corresponds to a different physical scenario of nonclassical interference. This indicates that this block diagonalization and consequent interpretation are not only fundamental but also universal features of multiphoton interferometry. We experimentally confirm our theory by recovering the full coincidence landscape of three arbitrarily distinguishable photons. We show that the theory can be applied to higher numbers of photons with an exemplary simulation of the nonclassical interference of five photons through a $9 \times 9$ network. Our approach thus provides a deeper understanding of the rich spectrum of multiphoton nonclassical interference. While passive schemes like BosonSampling benefit most from this approach, it applies analogously to pivotal building blocks of linear optical quantum computing [24,25] as crucial nondestructive two-qubit gates exploit ancillary photons and thus rely on multiphoton interference [26,27].

\section{METHODS}

\section{A. State generation}

A Ti:sapphire oscillator emitting 150 -fs pulses at $789 \mathrm{~nm}$ and a repetition rate of $80 \mathrm{MHz}$ is frequency doubled in a $\mathrm{LiB}_{3} \mathrm{O}_{5}$ (LBO) crystal (see Fig. 6 for a schematic of the experimental setup). The output power of this second harmonic generation can be controlled by a power regulation stage consisting of a half-wave plate (HWP) and a polarizing beam splitter (PBS) placed before the LBO crystal. The resulting emission at $394.5 \mathrm{~nm}$ is focused into a 2-mm-thick $\beta$ - $\mathrm{BaB}_{2} \mathrm{O}_{4}(\mathrm{BBO})$ crystal cut for degenerate noncollinear type-II down-conversion [28]. A compensation scheme consisting of HWPs and 1-mm-thick BBO

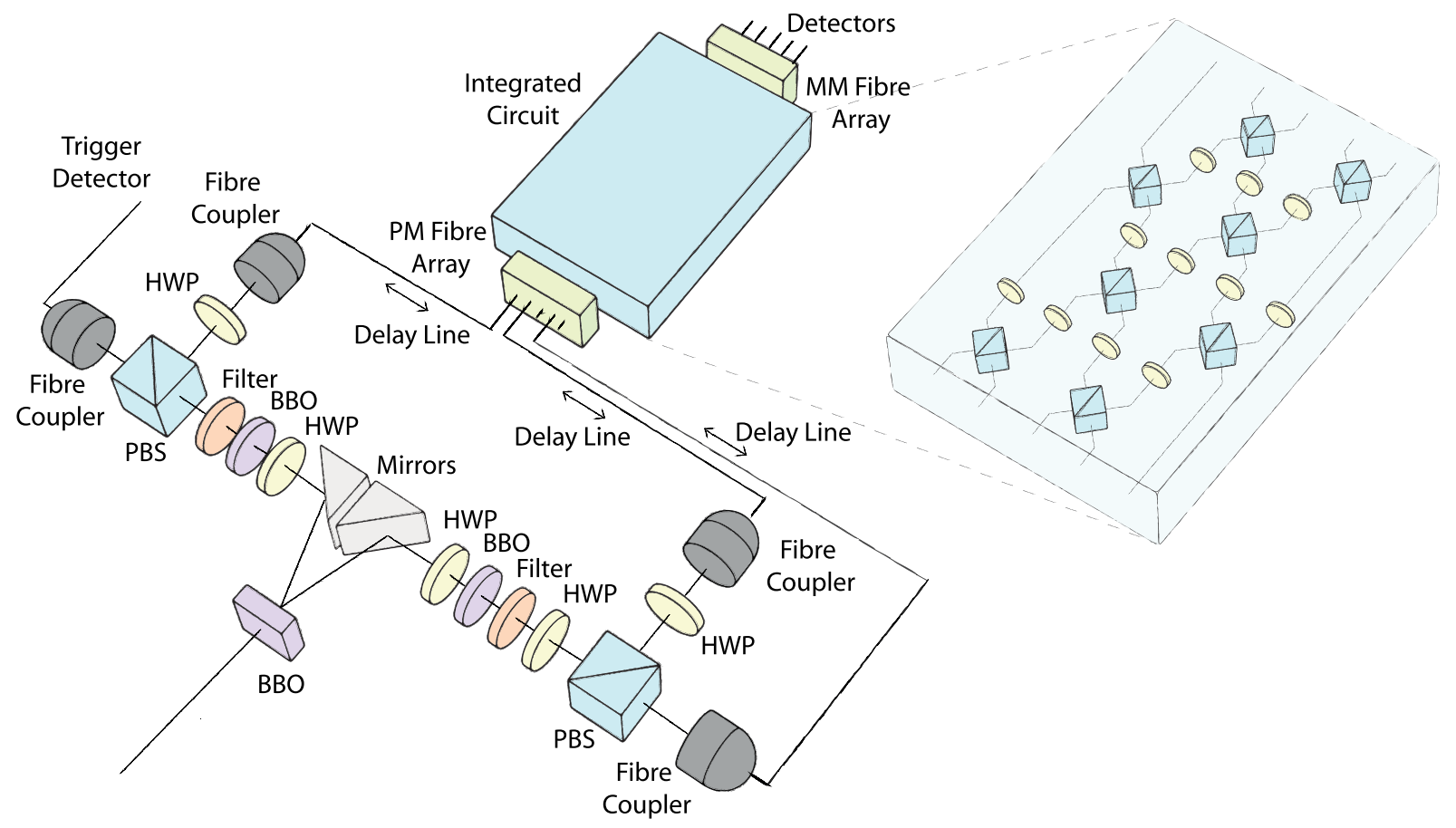

FIG. 6. Experimental setup. Four photons are generated via spontaneous parametric down-conversion and distributed to four spatial modes with two PBSs. A fourfold coincidence event consisting of three photons exiting the network and a trigger event postselects the desired input state. The delay lines allow us to tune the distinguishability and therefore the quantum interference of the three photons propagating through the waveguide. The integrated circuit is shown in a Mach-Zehnder decomposition and consists of eight beam splitters and 11 phase shifters. 
crystals is applied to counter temporal and spatial walk-off. The two spatial outputs of the down-converter pass through narrow band interference filters $\left(\lambda_{\mathrm{FWHM}}=3 \mathrm{~nm}\right)$ to achieve a coherence time greater than the birefringent walk-off due to group velocity mismatch in the crystal $\left(\left|v_{g_{e}}-v_{g_{o}}\right| \times\right.$ half-crystal thickness). Additionally, this renders the photons close to spectral indistinguishability. The down-conversion source is aligned to emit the maximally entangled Bell state $\left|\phi^{+}\right\rangle=(1 / \sqrt{2})(|H H\rangle+|V V\rangle)$ when pumped at 205-mW cw-equivalent pump power. The state is coupled into single-mode fibers (Nufern 780-HP) equipped with pedal-based polarization controllers to counter any stress-induced rotation of the polarization inside the fiber. Each of these spatial modes is then coupled to one input of a PBS while its other input is occupied with a vacuum state. The outputs pass HWPs and are subsequently coupled to four polarizationmaintaining (PM) fibers (Nufern PM780-HP). Temporal overlap is controlled by two motorized delay lines that exhibit a bidirectional repeatability of $\pm 1 \mu \mathrm{m}$. Temporal alignment precision is limited by other factors in the setup to approximately $\pm 5 \mu \mathrm{m}$ and is therefore within a precision of $2.5 \%$ of the coherence length of the photons. The polarization-maintaining fibers are mated to a singlemode fiber v-groove array (Nufern PM780-HP) with a pitch of $127 \mu \mathrm{m}$ and butt coupled to the integrated circuit. The coupling is controlled by a manual six-axis flexure stage and is stable within $5 \%$ of the total singlephoton counts over $12 \mathrm{~h}$. The output fiber array consists of a multimode (MM) v-groove array (GIF-625) and the photons are detected by single-photon avalanche photodiodes that are recorded with a home-built fieldprogrammable gate array logic. The coincidence time window is set to $3 \mathrm{~ns}$. In order to measure the six points of the coincidence landscapes, a three-photon input state is injected into the integrated network (see Appendix A 5 for further details). Therefore, the BBO is pumped with $\mathrm{cw}$-equivalent power of $700 \mathrm{~mW}$ and the ratio of the sixphoton emission over the desired four-photon emission is measured to be below $5 \%$.

\section{B. Integrated network fabrication}

The integrated photonic networks are fabricated using a femtosecond direct-write writing technology [29,30]. Laser pulses are focused $370 \mu \mathrm{m}$ below the surface of a high-purity fused silica wafer by a NA $=0.6$ objective. The 200-nJ pulses exhibit a pulse duration of 150 fs at $100 \mathrm{KHz}$ repetition rate and a central wavelength of $800 \mathrm{~nm}$. In order to write the individual waveguides, the wafer is translated with a speed of $6 \mathrm{~cm} / \mathrm{s}$. The waveguide modes exhibit a mode field diameter of $21.4 \times$ $17.2 \mu \mathrm{m}^{2}$ for a wavelength of $789 \mathrm{~nm}$ and a propagation loss of $0.3 \mathrm{~dB} / \mathrm{cm}$. This results in a coupling loss of $-3.5 \mathrm{~dB}$ with the type of input fibers used in this experiment. Coupling to the output array results in negligible loss due to the use of multimode fibers.

\section{ACKNOWLEDGMENTS}

The authors thank I. Dhand and J. Cotter for helpful discussions, M. Tomandl for assistance with the illustrations, and J. Nielsen and J. Kulp for computational assistance. M. T., S. E. S., and P. W. acknowledge support from the European Commission with the project EQuaMEmulators of Quantum Frustrated Magnetism (No. 323714), GRASP-Graphene-Based Single-Photon Nonlinear Optical Devices (No. 613024), PICQUE-Photonic Integrated Compound Quantum Encoding (No. 608062), QuILMIQuantum Integrated Light Matter Interface (No. 295293) and QUCHIP-Quantum Simulation on a Photonic Chip (No. 641039), the Vienna Center for Quantum Science and Technology (VCQ), and the Austrian Science Fund (FWF) with the projects PhoQuSi Photonic Quantum Simulators (Y585-N20) and the doctoral programme CoQuS Complex Quantum Systems, the Vienna Science and Technology Fund (WWTF) under Grant No. ICT12-041, and the Air Force Office of Scientific Research, Air Force Material Command, United States Air Force, under Grant No. FA8655-11-1-3004. B.C.S. acknowledges support from AITF (Alberta Innovates Technology Futures), NSERC (Natural Sciences and Engineering Research Council), and CIFAR (Canadian Institute for Advanced Research). The work of H. deG. is supported in part by NSERC of Canada. This material is also based on research supported in part by the Singapore National Research Foundation under NRF Award No. NRF-NRFF2013-01 (S.-H. T.). R. H., S. N., and A.S. acknowledge support from the German Ministry of Education and Research (Center for Innovation Competence programme, Grant No. 03Z1HN31), the Deutsche Forschungsgemeinschaft (Grant No. NO462/6-1), and the Thuringian Ministry for Education, Science and Culture (Research group Spacetime, Grant No. 11027-514).

\section{APPENDIX A SUPPLEMENTARY INFORMATION}

\section{Two-photon nonclassical interference}

Two photons injected into different inputs of an arbitrary beam splitter or a network built from arbitrary beam splitters and phase shifters will interfere nonclassically $[10,31]$. This input state can be expressed as

$$
\left|\Psi_{i n_{11}}\right\rangle=\left[\hat{A}_{1}^{\dagger}\left(\alpha_{1}\right) e^{i \omega_{1} \tau_{1}}\right]\left[\hat{A}_{2}^{\dagger}\left(\alpha_{2}\right) e^{i \omega_{2} \tau_{2}}\right]|0\rangle,
$$

with

$$
\hat{A}_{i}^{\dagger}\left(\alpha_{i}\right)=\int_{0}^{\infty} d \omega_{i} \alpha_{i}\left(\omega_{i}\right) \hat{a}_{i}^{\dagger}\left(\omega_{i}\right)
$$


for $A_{i}^{\dagger}\left(\alpha_{i}\right)$, a creation operator for a photon with spectral function

$$
\left|\alpha\left(\omega_{i}\right)\right|^{2}=\frac{1}{\sqrt{2 \pi} \sigma_{i}} \exp \left(-\frac{\left(\omega_{i}-\omega_{c, i}\right)^{2}}{2 \sigma_{i}^{2}}\right)
$$

centered at time $\tau_{i}$. The frequency-mode creation operators on the rhs of Eq. (A2) satisfy the commutator relation

$$
\left[\hat{a}_{i}(\omega), \hat{a}_{j}^{\dagger}\left(\omega^{\prime}\right)\right]=\delta_{i j} \delta\left(\omega-\omega^{\prime}\right) \mathbb{1},
$$

with $\mathbb{1}$ the identity operator. This commutation relation also defines the photons' symmetry under permutation operations. For two photons it is sufficient to define their relative temporal delay as $\Delta \tau=\tau_{1}-\tau_{2}$. Only in the case of ideal bosonic particles exhibiting no modal mismatch and perfect temporal overlap, i.e., $\Delta \tau=0$, does the rhs of Eq. (A4) become the well-known bosonic commutator relation describing perfect symmetry under exchange. When the two-photon input state [see Eq. (A1)] is mixed via a transformation matrix $B=U_{2 \times 2}$ and projected on an output where the two photons exit in different modes, the output probability becomes

$$
\begin{aligned}
P_{c}(\Delta \tau)= & \int d \omega_{1} \int d \omega_{2}\left|\left\langle\Psi_{i n_{11}}\left|\hat{B}^{\dagger} \hat{a}_{1}^{\dagger}\left(\omega_{1}\right) \hat{a}_{2}^{\dagger}\left(\omega_{2}\right)\right| 0\right\rangle\right|^{2} \\
= & \left(\begin{array}{c}
\operatorname{per}(B) \\
\operatorname{det}(B)
\end{array}\right) \dagger\left[\frac{1}{2}\left(\begin{array}{ll}
1 & 0 \\
0 & 1
\end{array}\right)+\frac{1}{2} \zeta e^{-\xi \Delta \tau^{2}}\left(\begin{array}{cc}
1 & 0 \\
0 & -1
\end{array}\right)\right] \\
& \times\left(\begin{array}{c}
\operatorname{per}(B) \\
\operatorname{det}(B)
\end{array}\right) \\
= & \boldsymbol{v}_{2}^{\dagger}\left[\hat{R}^{(2)}(\Delta \tau)\right] \boldsymbol{v}_{2},
\end{aligned}
$$

with

$$
\zeta=\frac{2 \sigma_{1} \sigma_{2}}{\sigma_{1}^{2}+\sigma_{2}^{2}} \exp \left(-\frac{\left(\omega_{c, 1}-\omega_{c, 2}\right)^{2}}{2\left(\sigma_{1}^{2}+\sigma_{2}^{2}\right)}\right), \quad \xi=\frac{\sigma_{1}^{2} \sigma_{2}^{2}}{\sigma_{1}^{2}+\sigma_{2}^{2}}
$$

denoting factors arising from the spectral overlap integral and

$$
\boldsymbol{v}_{2}=\frac{1}{\sqrt{2}}\left(\begin{array}{c}
\operatorname{per}(B) \\
\operatorname{det}(B)
\end{array}\right)
$$

the new basis vector constituted by matrix functions of the scattering submatrix $T$. As a second-order correlation effect, this nonclassical interference is dependent on the permutational symmetry of the interfering wave functions also reflected in the basis vector $\boldsymbol{v}_{\mathbf{2}}$. For the case of indistinguishable photons $\left(\omega_{c, 1}=\omega_{c, 2}, \sigma_{1}=\sigma_{2}\right.$, or $\left.\Delta \tau=0\right)$, the output probability is only proportional to the permanent. This is a function symmetric under permutation of rows of the transformation matrix arising in photon interferometry due to bosonic exchange symmetry. However, with loss of complete indistinguishability $\left(\omega_{c 1} \neq \omega_{c 2}, \quad \sigma_{1} \neq \sigma_{2}\right.$, and $\left.\Delta \tau \neq 0\right)$, Eq. (A6) becomes proportional to a combination of the determinant and the permanent. This is a consequence of the input state losing its symmetry under exchange. Equation (A7) decouples the influence of the interferometer from the influence of the input state. The latter is contained in the diagonal $2 \times 2$ rate matrix $\hat{R}^{(2)}(\Delta \tau)$, whereas the description of the interferometer is absorbed in the new basis vector $\boldsymbol{v}_{2}$. The two nonzero entries of the rate matrix, $\hat{R}_{11}^{(2)}$ and $\hat{R}_{22}^{(2)}$, are sufficient to reveal the nature of the nonclassical interference of two photons of arbitrary coherence. Where $\hat{R}_{11}^{(2)}$ quantifies the contribution from the permanent of the scattering submatrix, $\hat{R}_{22}^{(2)}$ quantifies the contribution from the determinant of the scattering submatrix. The output probability $P_{c}$ is recovered by calculating those matrix functions.

\section{Three-photon nonclassical interference}

Nonclassical interference of photons depends on indistinguishability of the interfering photons and transformations mixing the modes. Adding a third photon noticeably increases the complexity. An input state corresponding to three photons in three different transverse spatiotemporal modes can be described as

$$
\left|\Psi_{i n_{11}}\right\rangle=\left[A_{1}^{\dagger}\left(\alpha_{1}\right) e^{i \omega_{1} \tau_{1}}\right]\left[A_{2}^{\dagger}\left(\alpha_{2}\right) e^{i \omega_{2} \tau_{2}}\right]\left[A_{3}^{\dagger}\left(\alpha_{3}\right) e^{i \omega_{3} \tau_{3}}\right]|0\rangle .
$$

For three photons it is sufficient to define two relative temporal delays, $\Delta \tau_{1}=\tau_{1}-\tau_{2}$ and $\Delta \tau_{2}=\tau_{3}-\tau_{2}$. When this input state is transformed via a submatrix $T=U_{3 \times 3}$ and projected on an output where the three photons exit in different modes, the fully expanded output probability can be written as 


$$
\begin{aligned}
& P_{111}\left(\Delta \tau_{1}, \Delta \tau_{2}\right)=\int d \omega \int d \omega^{\prime} \int d \omega^{\prime \prime}\left|\left\langle\Psi_{i n_{111}}\left|\hat{T}^{\dagger} a_{1}^{\dagger}(\omega) a_{2}^{\dagger}\left(\omega^{\prime}\right) a_{3}^{\dagger}\left(\omega^{\prime \prime}\right)\right| 0\right\rangle\right|^{2} \\
& =\frac{1}{6}|\operatorname{det}(T)|^{2}+\frac{2}{9}\left|\operatorname{imm}\left(T_{132}\right)\right|^{2}+\frac{1}{9} \operatorname{imm}^{*}\left(T_{132}\right) \operatorname{imm}\left(T_{213}\right)+\frac{1}{9} \operatorname{imm}\left(T_{132}\right) \operatorname{imm} *\left(T_{213}\right) \\
& +\frac{2}{9}\left|\operatorname{imm}\left(T_{213}\right)\right|^{2}+\frac{2}{9}\left|\operatorname{imm}\left(T_{231}\right)\right|^{2}+\frac{2}{9}|\operatorname{imm}(T)|^{2}+\frac{1}{9} \operatorname{imm}\left(T_{231}\right) \operatorname{imm}^{*}(T) \\
& +\frac{1}{6}|\operatorname{per}(T)|^{2}+\frac{1}{9} \operatorname{imm}(T) \operatorname{imm}^{*}\left(T_{231}\right) \\
& +\zeta_{13} \exp \left(-2 \xi_{13}\left(\Delta \tau_{1}-\Delta \tau_{2}\right)^{2}\right)\left[-\frac{1}{6}|\operatorname{det}(T)|^{2}-\frac{2}{9} \operatorname{imm}(T) \operatorname{imm}^{*}\left(T_{132}\right)-\frac{1}{9} \operatorname{imm}(T) \operatorname{imm}^{*}\left(T_{213}\right)\right. \\
& -\frac{1}{9} \mathrm{imm}^{*}\left(T_{132}\right) \operatorname{imm}\left(T_{231}\right)+\frac{1}{9} \mathrm{imm}^{*}\left(T_{213}\right) \operatorname{imm}\left(T_{231}\right)-\frac{1}{9} \operatorname{imm}\left(T_{132}\right) \operatorname{imm}^{*}\left(T_{231}\right) \\
& \left.+\frac{1}{9} \operatorname{imm}\left(T_{213}\right) \operatorname{imm}^{*}\left(T_{231}\right)-\frac{2}{9} \operatorname{imm}\left(T_{132}\right) \operatorname{imm}^{*}(R)-\frac{1}{9} \operatorname{imm}\left(T_{213}\right) \operatorname{imm}^{*}(T)+\frac{1}{6}|\operatorname{per}(T)|^{2}\right] \\
& +\zeta_{12} \exp \left(-2 \xi_{12} \Delta \tau_{1}^{2}\right)\left[-\frac{1}{6}|\operatorname{det}(T)|^{2}+\frac{1}{9} \operatorname{imm}(T) \operatorname{imm}^{*}\left(T_{132}\right)+\frac{2}{9} \operatorname{imm}(T) \operatorname{imm}^{*}\left(T_{213}\right)\right. \\
& +\frac{2}{9} \mathrm{imm}^{*}\left(T_{132}\right) \operatorname{imm}\left(T_{231}\right)+\frac{1}{9} \mathrm{imm}^{*}\left(T_{213}\right) \operatorname{imm}\left(T_{231}\right)+\frac{2}{9} \operatorname{imm}\left(T_{132}\right) \operatorname{imm}^{*}\left(T_{231}\right) \\
& \left.+\frac{1}{9} \operatorname{imm}\left(T_{213}\right) \operatorname{imm}^{*}\left(T_{231}\right)+\frac{1}{9} \operatorname{imm}\left(T_{132}\right) \operatorname{imm}^{*}(T)+\frac{2}{9} \operatorname{imm}\left(T_{213}\right) \operatorname{imm}^{*}(T)+\frac{1}{6}|\operatorname{per}(T)|^{2}\right] \\
& +\zeta_{23} \exp \left(-2 \xi_{23} \Delta \tau_{2}^{2}\right)\left[-\frac{1}{6}|\operatorname{det}(T)|^{2}+\frac{1}{9} \operatorname{imm}(T) \operatorname{imm}^{*}\left(T_{132}\right)-\frac{1}{9} \operatorname{imm}(T) \operatorname{imm}^{*}\left(T_{213}\right)\right. \\
& -\frac{1}{9} \mathrm{imm}^{*}\left(T_{132}\right) \operatorname{imm}\left(T_{231}\right)-\frac{2}{9} \mathrm{imm}^{*}\left(T_{213}\right) \operatorname{imm}\left(T_{231}\right)-\frac{1}{9} \operatorname{imm}\left(T_{132}\right) \operatorname{imm}^{*}\left(T_{231}\right) \\
& \left.-\frac{2}{9} \operatorname{imm}\left(T_{213}\right) \operatorname{imm}^{*}\left(T_{231}\right)+\frac{1}{9} \operatorname{imm}\left(T_{132}\right) \operatorname{imm}^{*}(T)-\frac{1}{9} \operatorname{imm}\left(T_{213}\right) \operatorname{imm} *(T)+\frac{1}{6}|\operatorname{per}(T)|^{2}\right] \\
& +\zeta_{123} \exp \left(-I_{a}+i I_{s}\right)\left[\frac{1}{6}|\operatorname{det}(T)|^{2}-\frac{1}{9}\left|\operatorname{imm}\left(T_{132}\right)\right|^{2}-\frac{2}{9} \operatorname{imm}^{*}\left(T_{132}\right) \operatorname{imm}\left(T_{213}\right)\right. \\
& +\frac{1}{9} \operatorname{imm}\left(T_{132}\right) \operatorname{imm}^{*}\left(T_{213}\right)-\frac{1}{9}\left|\operatorname{imm}\left(T_{213}\right)\right|^{2}+\frac{1}{9} \operatorname{imm}(T) \operatorname{imm}^{*}\left(T_{231}\right)-\frac{1}{9}\left|\operatorname{imm}\left(T_{231}\right)\right|^{2} \\
& \left.-\frac{1}{9}|\operatorname{imm}(T)|^{2}-\frac{2}{9} \operatorname{imm}\left(T_{231}\right) \operatorname{imm} *(T)+\frac{1}{6}|\operatorname{per}(T)|^{2}\right] \\
& +\zeta_{123} \exp \left(-I_{a}-i I_{s}\right)\left[\frac{1}{6}|\operatorname{det}(T)|^{2}-\frac{1}{9}\left|\operatorname{imm}\left(T_{132}\right)\right|^{2}-\frac{2}{9} \operatorname{imm}\left(T_{132}\right) \operatorname{imm} *\left(T_{213}\right)\right. \\
& +\frac{1}{9} \mathrm{imm}^{*}\left(T_{132}\right) \operatorname{imm}\left(T_{213}\right)-\frac{1}{9}\left|\operatorname{imm}\left(T_{213}\right)\right|^{2}+\frac{1}{9} \mathrm{imm}^{*}(T) \operatorname{imm}\left(T_{231}\right)-\frac{1}{9}\left|\operatorname{imm}\left(T_{231}\right)\right|^{2} \\
& \left.-\frac{1}{9}|\operatorname{imm}(T)|^{2}-\frac{2}{9} \operatorname{imm}^{*}\left(T_{231}\right) \operatorname{imm}(T)+\frac{1}{6}|\operatorname{per}(T)|^{2}\right] \text {, }
\end{aligned}
$$

with

$$
\begin{aligned}
\zeta_{123} & =\sqrt{\zeta_{12} \zeta_{23} \zeta_{13}}, \\
I_{a} & \equiv I_{a}\left(\Delta \tau_{1}, \Delta \tau_{2}\right)=-\left(\Delta \tau_{1}\right)^{2} \frac{\xi_{12}}{2}-\left(\Delta \tau_{1}-\Delta \tau_{2}\right)^{2} \frac{\xi_{13}}{2}-\left(\Delta \tau_{2}\right)^{2} \frac{\xi_{23}}{2} \\
I_{s} & \equiv I_{s}\left(\Delta \tau_{1}, \Delta \tau_{2}\right)=\Delta \tau_{1} \nu_{12}-\left(\Delta \tau_{1}-\Delta \tau_{2}\right) \nu_{13}-\Delta \tau_{2} \nu_{23}, \\
\zeta_{i j} & =\frac{2 \sigma_{i} \sigma_{j}}{\sigma_{i}^{2}+\sigma_{j}^{2}} \exp \left(-\frac{\left(\omega_{c, i}-\omega_{c, j}\right)^{2}}{2\left(\sigma_{i}^{2}+\sigma_{j}^{2}\right)}\right)
\end{aligned}
$$




$$
\xi_{i j}=\frac{2 \sigma_{i}^{2} \sigma_{j}^{2}}{\sigma_{i}^{2}+\sigma_{j}^{2}}, \quad \nu_{i j}=\frac{\omega_{c, i} \sigma_{j}^{2}+\omega_{c, j} \sigma_{i}^{2}}{\sigma_{i}^{2}+\sigma_{j}^{2}} .
$$

The subscripts denote the mode labels for the submatrix $T . T_{i j k}$ is the matrix $T$ with the rows permuted according to $1 \rightarrow i$, $2 \rightarrow j$, and $3 \rightarrow k$.

For a more elegant expression, Eq. (A12) can be simplified introducting six matrices, $\mathbb{1}, \rho_{12}, \rho_{13}, \rho_{23}, \rho_{123}$, and $\rho_{132}$ :

$$
\begin{aligned}
P_{111}\left(\Delta \tau_{1}, \Delta \tau_{2}\right)= & \left(\hat{P} \hat{S} \boldsymbol{v}_{\mathbf{3}}\right)^{\dagger}\left[\mathbb{1}+\rho_{12} \zeta_{12} e^{-\xi_{12} \Delta \tau_{1}^{2}}+\rho_{23} \zeta_{23} e^{-\xi_{23} \Delta \tau_{2}^{2}}+\rho_{13} \zeta_{13} e^{-\xi_{13}\left(\Delta \tau_{1}-\Delta \tau_{2}\right)^{2}}\right. \\
& \left.+\zeta_{123}\left(\rho_{132} e^{\xi_{123}^{*}\left(\Delta \tau_{1}, \Delta \tau_{2}\right)}+\rho_{123} e^{\xi_{123}\left(\Delta \tau_{1}, \Delta \tau_{2}\right)}\right)\right]\left(\hat{P} \hat{S} \boldsymbol{v}_{\mathbf{3}}\right) \\
= & \boldsymbol{v}_{3}^{\dagger}\left[\hat{R}^{(3)}\left(\Delta \tau_{1}, \Delta \tau_{2}\right)\right] \boldsymbol{v}_{\mathbf{3}},
\end{aligned}
$$

where

$$
\xi_{123}\left(\Delta \tau_{1}, \Delta \tau_{2}\right)=I_{a}+i I_{s}
$$

The vector $\hat{P} \hat{S} \boldsymbol{v}_{\mathbf{3}}$ contains all the immanants and the determinant and permanent of $T$ :

$$
\hat{P} \hat{S} v_{3} \equiv\left(\begin{array}{c}
\frac{1}{\sqrt{6}} \operatorname{per}(T) \\
\frac{1}{\sqrt{6}} \operatorname{det}(T) \\
\frac{1}{2 \sqrt{3}} \operatorname{imm}(T)+\frac{1}{2 \sqrt{3}} \operatorname{imm}\left(T_{213}\right) \\
\frac{1}{6} \operatorname{imm}(T)-\frac{1}{3} \operatorname{imm}\left(T_{132}\right)-\frac{1}{6} \operatorname{imm}\left(T_{213}\right)+\frac{1}{3} \operatorname{imm}\left(T_{312}\right) \\
\frac{1}{6} \operatorname{imm}(T)+\frac{1}{3} \operatorname{imm}\left(T_{132}\right)+\frac{1}{6} \operatorname{imm}\left(T_{213}\right)+\frac{1}{3} \operatorname{imm}\left(T_{312}\right) \\
-\frac{1}{2 \sqrt{3}} \operatorname{imm}(T)+\frac{1}{2 \sqrt{3}} \operatorname{imm}\left(T_{213}\right)
\end{array}\right)
$$

with

$$
\begin{aligned}
\boldsymbol{v}_{3}=\left(\begin{array}{c}
\operatorname{per}(T) \\
\operatorname{imm}(T) \\
\operatorname{imm}\left(T_{132}\right) \\
\operatorname{imm}\left(T_{213}\right) \\
\operatorname{imm}\left(T_{312}\right) \\
\operatorname{det}(T)
\end{array}\right), \\
\hat{P}=\left(\begin{array}{cccccc}
\frac{1}{\sqrt{6}} & \frac{1}{\sqrt{6}} & \frac{1}{\sqrt{6}} & \frac{1}{\sqrt{6}} & \frac{1}{\sqrt{6}} & \frac{1}{\sqrt{6}} \\
\frac{1}{\sqrt{6}} & -\frac{1}{\sqrt{6}} & -\frac{1}{\sqrt{6}} & \frac{1}{\sqrt{6}} & \frac{1}{\sqrt{6}} & -\frac{1}{\sqrt{6}} \\
\frac{1}{\sqrt{3}} & -\frac{1}{2 \sqrt{3}} & \frac{1}{\sqrt{3}} & -\frac{1}{2 \sqrt{3}} & -\frac{1}{2 \sqrt{3}} & -\frac{1}{2 \sqrt{3}} \\
0 & -\frac{1}{2} & 0 & -\frac{1}{2} & \frac{1}{2} & \frac{1}{2} \\
0 & \frac{1}{2} & 0 & -\frac{1}{2} & \frac{1}{2} & -\frac{1}{2} \\
-\frac{1}{\sqrt{3}} & -\frac{1}{2 \sqrt{3}} & \frac{1}{\sqrt{3}} & \frac{1}{2 \sqrt{3}} & \frac{1}{2 \sqrt{3}} & -\frac{1}{2 \sqrt{3}}
\end{array}\right), \quad \hat{S}=\left(\begin{array}{cccccccc}
\frac{1}{6} & \frac{1}{3} & 0 & 0 & 0 & \frac{1}{6} \\
\frac{1}{6} & 0 & \frac{1}{3} & 0 & 0 & -\frac{1}{6} \\
\frac{1}{6} & 0 & 0 & \frac{1}{3} & 0 & -\frac{1}{6} \\
\frac{1}{6} & -\frac{1}{3} & 0 & 0 & -\frac{1}{3} & \frac{1}{6} \\
\frac{1}{6} & 0 & 0 & 0 & \frac{1}{3} & \frac{1}{6} \\
\frac{1}{6} & 0 & -\frac{1}{3} & -\frac{1}{3} & 0 & -\frac{1}{6}
\end{array}\right) .
\end{aligned}
$$

Here, $\hat{P}$ is a basis transformation and $\hat{S}$ is a matrix mapping matrix elements to matrix functions. The six matrices $\rho$ are, in fact, permutation matrices reduced to the block-diagonal form of Eq. (10).

Equation (A15) describes the same features as Eq. (A12) but highlights the permutational options for three photons. It is given in a maximally decoupled basis, which allows for a compact notation. The terms originating from the overlap integrals ( $\zeta$ terms and $\xi$ terms) contain all the information on the physical properties of the interfering photons. The effect of the permutation symmetry of the photons is included in the permutation matrices $\rho$. Equation (A16) features an even further compressed notation and allows for an elegant interpretation. Whereas the block-diagonal $6 \times 6$ rate matrix 
$\hat{R}^{(3)}\left(\Delta \tau_{1}, \Delta \tau_{2}\right)$ contains all the information on the permutational symmetry and nonclassical interference itself, the basis vector $\boldsymbol{v}_{\mathbf{3}}$ contains the information on the interferometer. As alluded in Sec. IV two entries of this rate matrix are sufficient for an interpretation. $F_{\text {per }}=\hat{R}_{11}^{(3)}\left(\Delta \tau_{1}, \Delta \tau_{2}\right)$ quantifies the fraction of the output probability distribution proportional to the permanent and $F_{\text {det }}=\hat{R}_{66}^{(3)}\left(\Delta \tau_{1}, \Delta \tau_{2}\right)$ to the determinant of the submatrix $T$. The contribution proportional to immanants can also be explicitly calculated. When interested in only their overall contribution, this is given as $F_{\text {imm }}=1-F_{\text {per }}-F_{\text {det }}$. In the extremal case when all the photons are indistinguishable, i.e.,

$\omega_{c, 1}=\omega_{c, 2}=\omega_{c, 3}=\omega_{c}, \quad \sigma_{1}=\sigma_{2}=\sigma_{3}=\sigma$,

$\Delta \tau_{1}=\Delta \tau_{2}=0$

we have $\zeta_{i j}=1, \xi_{i j}=\sigma^{2}$, and $\nu_{i j}=\omega$, so the output probability reduces from a superposition of 60 terms to just $P_{111} \rightarrow|\operatorname{per}(T)|^{2}$. The rate matrix for the case of completely indistinguishable photons, $\hat{R}^{(3)}\left(\Delta \tau_{1}=0, \Delta \tau_{2}=0\right)$, describing the perfect quantum interference, and the rate matrix for the case of completely distinguishable photons, $\hat{R}^{(3)}\left(\Delta \tau_{1}=\infty, \Delta \tau_{2}=-\infty\right)$, describing the classical case, are given as

$$
\begin{aligned}
\hat{R}^{(3)}\left(\Delta \tau_{1}=0, \Delta \tau_{2}=0\right) & \left(\begin{array}{cccccc}
1 & 0 & 0 & 0 & 0 & 0 \\
0 & 0 & 0 & 0 & 0 & 0 \\
0 & 0 & 0 & 0 & 0 & 0 \\
0 & 0 & 0 & 0 & 0 & 0 \\
0 & 0 & 0 & 0 & 0 & 0 \\
0 & 0 & 0 & 0 & 0 & 0
\end{array}\right), \\
\hat{R}^{(3)}\left(\Delta \tau_{1}=\infty, \Delta \tau_{2}=-\infty\right) & =\left(\begin{array}{cccccc}
\frac{1}{6} & 0 & 0 & 0 & 0 & 0 \\
0 & \frac{2}{9} & 0 & 0 & \frac{1}{9} & 0 \\
0 & 0 & \frac{2}{9} & \frac{1}{9} & 0 & 0 \\
0 & 0 & \frac{1}{9} & \frac{2}{9} & 0 & 0 \\
0 & \frac{1}{9} & 0 & 0 & \frac{2}{9} & 0 \\
0 & 0 & 0 & 0 & 0 & \frac{1}{6}
\end{array}\right) .
\end{aligned}
$$

\section{Matrix reconstruction}

The fabrication of integrated photonic networks using a femtosecond-laser direct-writing technology works with high precision and high stability. Discrete unitary operators acting on modes can be realized solely from beam splitters and phase shifters [32]. These networks are arranged like cascaded Mach-Zehnder interferometers, shown in Fig. 7. Notably though, even advanced writing precision can introduce small deviations from the initially targeted values of individual elements. In our case this writing precision is limited to around $50 \mathrm{~nm}$ over the whole length of the waveguide (in this experiment, $10 \mathrm{~cm}$ ). In a cascaded interferometric arrangement small deviations of individual elements may add up to a noticeable deviation in the overall transformation. The splitting ratio of individual directional couplers is set by their mode separation and coupling length. Both characteristic variables are 3 orders of magnitude bigger than the positioning precision and therefore unaffected by it. Unfortunately, small length fluctuations due to the positioning precision can introduce unintended phase shifts. In the worst case, i.e., a phase shifter spanning the whole length of a waveguide, the resultant phase shifts can even reach $\pi / 8$. The layout used for the interferometric networks reported here (see Fig. 7) circumvents this worst case. Even if the unintended phase shifts are decreased by a factor of 3 at least, their influence needs to be evaluated and the actually implemented unitary needs to be reconstructed. The characterization procedure we use builds on the one introduced in Refs. [33,34]. Two-photon states from a down-conversion source are injected into different modes of the optical network to be characterized. This in situ method allows for a characterization with states having the same physical properties, e.g., frequency and spectral shape, as used later in the experiment.

\section{a. Estimating the visibilities of submatrices}

We assume the optical interferometer can be described by a $5 \times 5$ unitary matrix and we reconstruct its

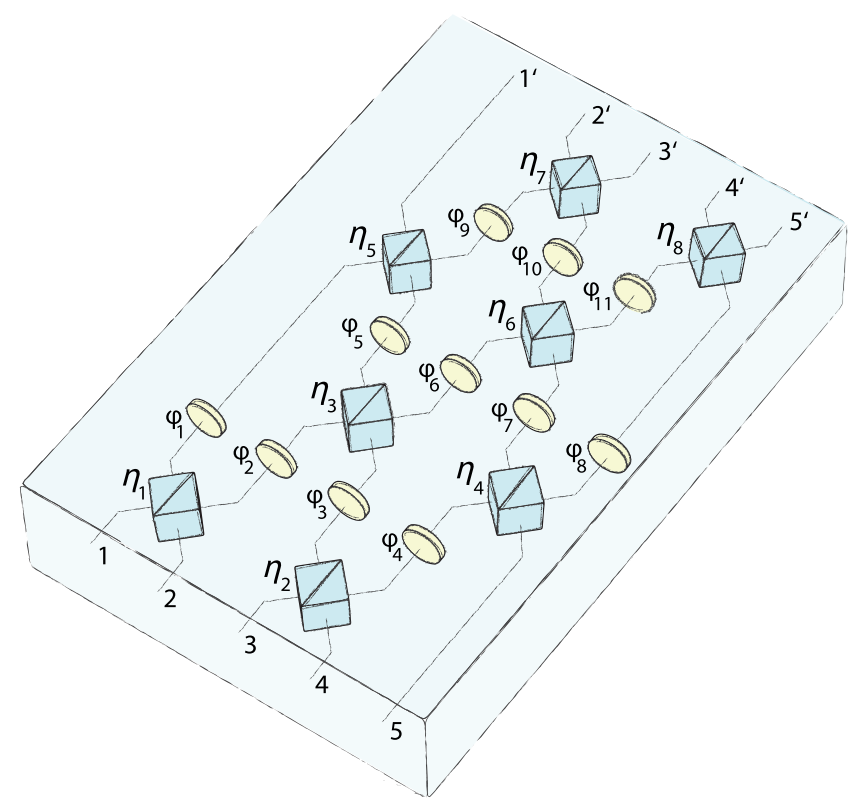

FIG. 7. Integrated photonic network. Schematic drawing of the optical network. The circuit consists of eight directional couplers $\left(\eta_{1}, \ldots, \eta_{8}\right), 11$ phase shifters $\left(\phi_{1}, \ldots, \phi_{11}\right)$, five input modes $(1, \ldots, 5)$, as well as five output modes $\left(1^{\prime}, \ldots, 5^{\prime}\right)$. To allow coupling to the waveguide with standard fiber arrays, the input and output modes are separated $127 \mu \mathrm{m}$ and the total length of the chip is $10 \mathrm{~cm}$. 
transformation via visibilities measured by injecting two photons into any combination of two of its five inputs. The visibility for two photons entering input modes $i, j$ and exiting in the output modes $k, l$ can be calculated from the $2 \times 2$ submatrix $U_{i, j, k, l}$. For five input and output modes this results in $\left(\begin{array}{l}5 \\ 2\end{array}\right) \times\left(\begin{array}{l}5 \\ 2\end{array}\right)=100$ possibilities. Owing to the structure of the interferometer (see Fig. 7), a photon injected into port 5 cannot exit from output $1^{\prime}$. This leads to a visibility of zero for the four input pairs $i j=$ $15,25,35,45$ and the output pairs $k l=15,25,35,45$. These visibilities are omitted from this reconstruction algorithm, so the unitary transformation is reconstructed from 84 nonzero visibilities.

Our interferometric network consists of eight beam splitters and 11 phase shifters. Each beam splitter implements a $\mathrm{SU}(2)$ transformation with matrix representation:

$$
\left(\begin{array}{cc}
\cos \frac{\beta}{2} & i \sin \frac{\beta}{2} \\
i \sin \frac{\beta}{2} & \cos \frac{\beta}{2}
\end{array}\right),
$$

where $\beta$ is the Euler angle associated with the transmittivity $\eta$ via the relationship $\eta=\cos ^{2}(\beta / 2)$. Note that in Eq. (A21) the beam splitter also implements a relative phase shift of $\pi$ between the first and second mode.

The 11 phase shifters produce additional phases in their respective modes. Each phase shifter has a matrix representation of

$$
\left(\begin{array}{cc}
e^{i \alpha_{1}} & 0 \\
0 & e^{i \alpha_{2}}
\end{array}\right)
$$

with $\alpha_{i}$ the phase shift in mode $i$.

The spectral shape of the photons is measured with a single-photon spectrometer (Ocean Optics QE6500) and to a good approximation is of Gaussian shape. Such Gaussians are defined by only two parameters, namely, their central frequency and the variance, which for the $i$ th photon of the input pair is given by Eq. (A3), and expressed here as

$$
\left|\phi_{i}(\omega)\right|^{2}=\frac{1}{\sqrt{2 \pi} \sigma_{i}} \exp \left(-\frac{\left(\omega-\omega_{c, i}\right)^{2}}{2 \sigma_{i}^{2}}\right), \quad i=1,2 .
$$

Assuming both photons exhibit identical spectral function, i.e., $\left|\phi_{1}(\omega)\right|^{2}=\left|\phi_{2}(\omega)\right|^{2}$, and the detectors are modeled by the detection positive-operator valued measure (POVM) with

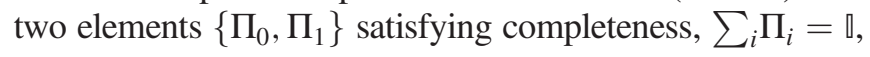

$$
\Pi_{1}=\int d \omega a^{\dagger}(\omega)|0\rangle\langle 0| a(\omega), \quad \Pi_{0}=\rrbracket-\Pi_{1},
$$

then the visibility is

$$
V=-\frac{h_{1} h_{2}^{*}+h_{1}^{*} h_{2}}{\left|h_{1}\right|^{2}+\left|h_{2}\right|^{2}}
$$

with

$$
h_{1}=U_{i, j, k, l}^{11} U_{i, j, k, l}^{22}, \quad h_{2}=U_{i, j, k, l}^{12} U_{i, j, k, l}^{21},
$$

and $U_{i, j, k, l}^{a, b}$ denotes the element in the $a$ th row and $b$ th column of the matrix $U_{i, j, k, l}$. In an experiment the two photons will always have slightly different spectral functions whose mismatch needs to be accounted for. The central wavelengths and spectral bandwidths of the photons used in this characterization measurement are $\lambda_{c, 1}=789.05 \mathrm{~nm}, \Delta \lambda_{1}=2.9 \mathrm{~nm}$ and $\lambda_{c, 2}=788.60 \mathrm{~nm}, \Delta \lambda_{2}=2.9 \mathrm{~nm}$, respectively. The coincidence counts $N_{c}$ as a function of time delay $t$ and spectral mode mismatch are

$$
\begin{aligned}
N_{c}(t)= & (1+T * t)\left[Y_{0}+A \frac{2 \sigma_{1} \sigma_{2}}{\sigma_{1}^{2}+\sigma_{2}^{2}}\right. \\
& \times \exp \left(-\frac{\left(\omega_{c, 1}-\omega_{c, 2}\right)^{2}+4 \sigma_{1}^{2} \sigma_{2}^{2}\left(t-t_{c}\right)^{2}}{2\left(\sigma_{1}^{2}+\sigma_{2}^{2}\right)}\right) \\
& \left.-\left(H O_{1}+H O_{2}-d\right)\right],
\end{aligned}
$$

where $Y_{0}, A, t_{c}$, and $T$ are parameters to be fitted to the experimental data. The experimental data for a given inputoutput combination $i, j, k, l$ is typically recorded for 30 increments with a step width of $66 \mathrm{fs}$ and integrated over $800 \mathrm{~s}$ each step. The coincidences are readout by a fieldprogrammable gate array logic. As individual delays are set by translating a fiber coupler with a motorized screw (Newport LTA-HL), there can be a small drift in coupling efficiency over the whole delay range of $2000 \mathrm{fs}$. Without this drift, the background of the visibility would be a horizontal straight line. For drifts smaller than 5\% of the two-photon flux, the drift is in good approximation linear and can be modeled with an additional parameter $T$. The positioning precision of the delay lines is limited to approximately $\pm 5 \mu \mathrm{m}$, which is within $2.5 \%$ of the coherence time of the interfering photons. When the two-photon input state is generated via down-conversion pumped by a pulsed laser system, higher-order emission can lead to unwanted contribution to the input state. The first higher order, which is a fourfold emission, causes a small contribution of two photons in each input mode during the characterization of a $2 \times 2$ submatrix. This can add a constant background to the twofold coincidences in the following scenario: two photons in one input mode are lost and the two photons in the other input mode leave the network in different output ports. We measure such contributions by blocking one of the two input modes and recording the two-photon coincidences at the output. These signals are labeled $\mathrm{HO}_{1}$ and $\mathrm{HO}_{2}$, respectively, and subtracted from the data. The background coincidence rate $d$ may be interpreted as a contribution to $N_{c}$ stemming from dark counts due to electrical noise and background light. This rate $d$ is also present in $\mathrm{HO}_{1}$ and $\mathrm{HO}_{2}$. Therefore, it has to be added to Eq. (A27) to account for all unwanted coincidences 
only once. The error for the raw data is verified to be Poissonian. For the data processing, the error of the higherorder term $\left(\mathrm{HO}_{1}+\mathrm{HO}_{2}-d\right)$ and the abscissa error caused by the limited alignment precision of the delay lines need to be taken into account additionally. These errors provide weighting in the minimization algorithm and influence the standard errors of the fitted parameters. The visibility,

$$
V=1-\frac{Y_{0}+A}{Y_{0}}
$$

is finally calculated from the parameters $Y_{0}$ and $A$, whereas the width of the dip or peak is fixed by the spectral function of the two photons. Only 84 out of 100 visibilities are nonzero, and their value $\left\{V_{i}^{\text {(expt) }}, i=1, \ldots, 84\right\}$ and standard deviation $\left\{\sigma_{i}, i=1, \ldots, 84\right\}$ are extracted via the procedure outlined above. The resultant data fits with theory exhibit $\chi_{\text {red }}^{2}=1.74$ [35]. An example for one of the 84 data sets is shown in Fig. 8.

\section{b. Parameter estimation and reconstruction of the unitary matrix}

A unitary transformation of a linear optical network can be reconstructed from single-photon transmission probabilities and two-photon interference visibilities [34]. A technique using coherent states [36] follows a similar approach. Both techniques reconstruct the unitary description in a dephased representation where the singlephoton or single-input coherent state data are used to estimate the real parts of the matrix entries. The imaginary parts of the matrix-entries are reconstructed from the twophoton interference visibilities or directly from the relative phase shifts. When the layout and initially targeted parameters of the building blocks (see Fig. 7) of the interferometric network are known, their actual parameters can be fitted alternatively. Our technique uses an overcomplete set of visibilities, and the parameters of the interferometer that give an optimal fit to the experimentally measured visibilities are obtained using a least-squares optimization weighted with the standard errors of the experimental visibilities (see Appendix A 3 a for details). Eight of the 19 parameters are transmittivities, $\beta_{1}, \beta_{2}, \ldots \beta_{8}$, and 11 are phases, $\phi_{1}, \phi_{2}, \ldots \phi_{11}$. To find the best-fit set of parameters, the data are processed with a Matlab program that uses fmincon to minimize the function $V_{\text {opt }}$,

$$
V_{\mathrm{opt}}=\sum_{i=1}^{84} \frac{\left(V_{i}^{(\mathrm{expt})}-V_{i}^{(\mathrm{th})}\right)^{2}}{\sigma_{i}^{2} \Gamma},
$$

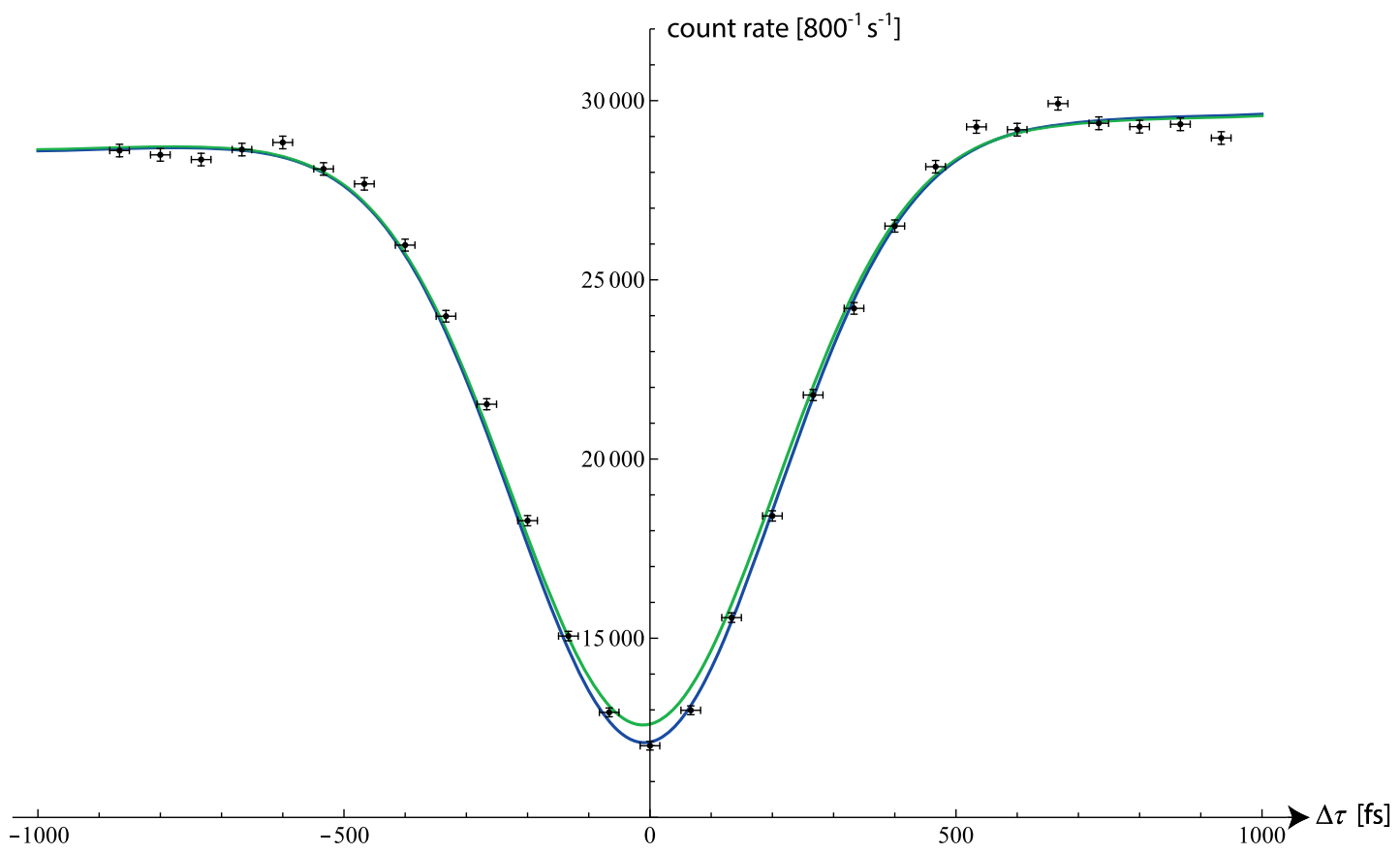

FIG. 8. Example for one data set used for the reconstruction of $U_{5}$. The best fit of Eq. (A27) to the data set is shown in blue. Here, the visibility is calculated from the best fit parameters $Y_{0}$ and $A$. The reduced $\chi^{2}$ resulting from the fit shown in blue is $\chi_{\text {blue }}^{2}=2.02$. Fluctuations in the count rate for values of $|\Delta \tau|>500$ fs drive the reduced $\chi^{2}$ away from 1 . These fluctuations can be interpreted as the random noise background in the lab and the increased reduced $\chi^{2}$ reflects that. However, the precision of the fitted parameters $Y_{0}$ and $A$ and ultimately the extracted visibility $V$ is only marginally affected by these fluctuations. The $5 \times 5$ unitary description of the interferometric network is reconstructed from 84 of these visibilities. The curve in green, $N_{c}^{(\text {th })}(t)$ [see Eq. (A33)] is calculated from four matrix entries of the reconstructed unitary and results in an overlap with the data of $\chi_{\text {green }}^{2}=2.70$. The agreement of these two curves and corresponding reduced $\chi^{2}$ 's is a qualitative measure for the precision of the reconstruction. 
where $V_{i}^{(\text {th })}$ is the theoretical value of the visibility calculated from our special unitary model of the interferometer using Eq. (A25) for the $i$ th data set, and $\Gamma$ is a constant value equal to (number of data sets in visibilitiesnumber of parameters -1$)=2522-19-188-1=2314$. Equation (A29) looks similar to a reduced $\chi^{2}$ but has to be interpreted differently. A value close to 0 is desirable and indicates good agreement between experimentally extracted and theoretically predicted visibilities. In our case, the result is $V_{\text {opt }}=0.351$.

The $5 \times 5$ reconstructed matrix $U_{5}$ using the procedure outlined above is

$$
U_{5}=\left(\begin{array}{ccccc}
0.0320-0.3370 i & 0.07239+0.8203 i & -0.2780-0.1060 i & 0.1228-0.3220 i & 0 \\
0.0114+0.2751 i & -0.3863+0.1860 i & -0.1353+0.2073 i & -0.7842-0.1502 i & 0.0124-0.2036 i \\
-0.7757-0.2328 i & -0.2937+0.0018 i & -0.2677-0.0162 i & 0.0267+0.3517 i & -0.2476-0.0151 i \\
0.1444-0.2611 i & -0.1518-0.0840 i & -0.1392+0.0839 i & -0.1327-0.0092 i & 0.0203+0.8449 i \\
0.2225+0.1231 i & 0.0715-0.1293 i & -0.7929-0.0268 i & 0.0871+0.3067 i & 0.4123-0.1121 i
\end{array}\right)
$$

\section{Quality of the reconstructed description}

Using this matrix, the probability of coincidence counts $P_{11}^{(\text {th })}$ can be predicted for any two-photon inputs and outputs. For the inputs $i$ and $j, i<j$, and outputs $k$ and $l, k<l$, this reads as

$$
\begin{aligned}
P_{11}^{(\mathrm{th})}\left(t-t_{c}\right)= & \left|U_{5}^{k i} U_{5}^{j l}\right|^{2}+\left|U_{5}^{l i} U_{5}^{k j}\right|^{2} \\
& +\left(U_{5}^{l i} U_{5}^{k j} U_{5}^{k i *} U_{5}^{j l *}\right. \\
& \left.+U_{5}^{l i *} U_{5}^{k j *} U_{5}^{k i} U_{5}^{j l}\right) f\left(t-t_{c}\right),
\end{aligned}
$$

where

$$
\begin{aligned}
f(t) \equiv & {\left[2 \sigma_{1} \sigma_{2} /\left(\sigma_{1}^{2}+\sigma_{2}^{2}\right)\right] } \\
& \times \exp \left(-\frac{\left(\omega_{c, 1}-\omega_{c, 2}\right)^{2}+4 \sigma_{1}^{2} \sigma_{2}^{2} t^{2}}{2\left(\sigma_{1}^{2}+\sigma_{2}^{2}\right)}\right),
\end{aligned}
$$

and $U_{5}^{a b}$ is the element in the $a$ th row and $b$ th column of $U_{5}$. The actual coincidence count is then

$$
N_{c}^{(\text {th })}(t)=N_{0}(1+T) P_{11}^{(\text {th })}\left(t-t_{c}\right),
$$

where $N_{0}, t_{c}$, and $T$ are parameters used to find the best fit to the experimental data. The exact $\chi_{\text {red }}^{2}$ is calculated using

$$
\chi_{\text {red }}^{2}=\sum_{i=1}^{m} \frac{\left(N_{c, i}^{(\text {expt })}-N_{c, i}^{(\mathrm{th})}\right)^{2}}{\nu \epsilon_{i}^{2}},
$$

where $m=3030, \nu=m-20-100-1=2909, \epsilon_{i}$ is the error for the corresponding data point, and $N_{c, i}^{(\text {expt })}$ denotes the experimental data corrected for higher-order emissions. The sum is taken over the data set and the index labels the data. The obtained $\chi_{\text {red }}^{2}$ between the data and the predicted coincidence counts using $U_{5}$ is

$$
\chi_{\text {red }}^{2}=2.086
$$

This value should be compared to reduced $\chi_{\text {expt }}^{2}=1.74$ obtained by fitting the primary data to extract the 84 visibilities in the beginning (see Appendix A 3 a). The difference between those two reduced $\chi^{2}$ 's can be attributed

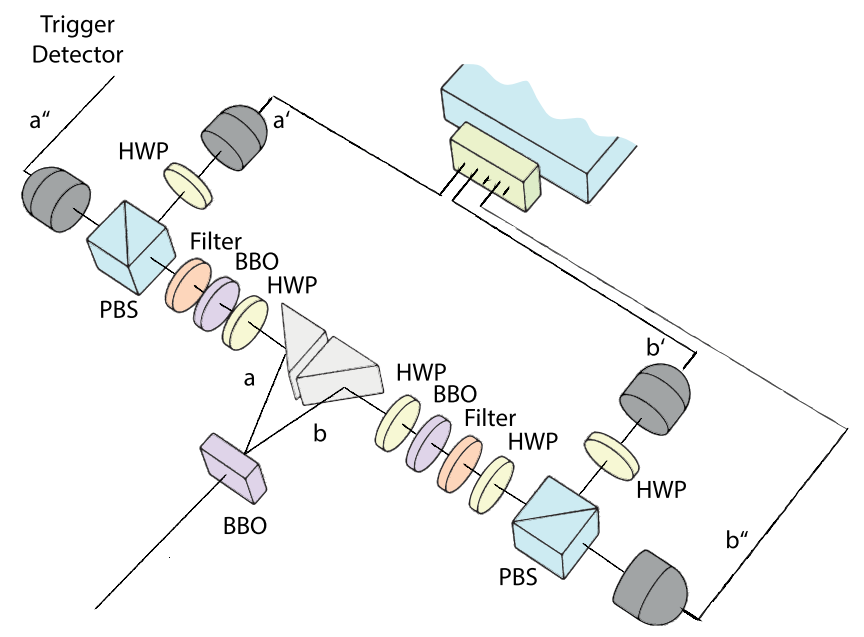

FIG. 9. State generation. A pump beam is focused into a 2-mm $\beta-\mathrm{BaB}_{2} \mathrm{O}_{2}$ crystal cut for noncollinear, degenerate, type-II downconversion. The generated state is emitted into the spatial modes $a$ and $b$. A compensation scheme consisting of half-wave plates and 1-mm-thick BBO crystals is applied for countering temporal and spatial walk-off. Narrow band interference filters $\left(\lambda_{\mathrm{FWHM}}=3 \mathrm{~nm}\right)$ are applied to increase the temporal coherence of the photons and render them close to spectral indistinguishability. The modes $a$ and $b$ are subsequently split by polarizing beam splitter cubes and two half-wave plates in their reflected ports are set to $45^{\circ}$ to ensure the same polarization in all four output modes $\left(a^{\prime \prime}, a^{\prime}, b^{\prime}\right.$, and $\left.b^{\prime \prime}\right)$. With this scheme, three indistinguishable photons in modes $a^{\prime}, b^{\prime}$, and $b^{\prime \prime}$ each can be heralded from a fourfold emission by a successful trigger event in mode $a^{\prime \prime}$. 
to accuracy of the reconstructed unitary matrix $U_{5}$. While fluctuations in the count rate for values of $|\Delta \tau|>500 \mathrm{fs}$ drive both reduced $\chi^{2}$ away from 1 , the difference between the two reduced $\chi^{2}$ is relatively small $(\approx 0.35)$. An example for one of the 100 data sets is shown in Fig. 8.

\section{State generation}

We use an 80-MHz Ti:sapphire oscillator emitting 150-fs pulses at a wavelength of $789 \mathrm{~nm}$, which get frequency doubled via a $\mathrm{LiB}_{3} \mathrm{O}_{5}$. The up-converted beam is focused into a 2-mm-thick $\beta$ - $\mathrm{BaB}_{2} \mathrm{O}_{2}$ crystal cut for degenerate noncollinear type-II spontaneous parametric downconversion. To achieve near-spectral indistinguishability and enhance temporal coherence of the down-converted wave packets, the photons are filtered by $\lambda_{\mathrm{FWHM}}=3 \mathrm{~nm}$ interference filters. The source is aligned to emit the maximally entangled state

$$
\left|\phi^{+}\right\rangle=\frac{1}{\sqrt{2}}\left(|H\rangle_{a}|H\rangle_{b}+|V\rangle_{a}|V\rangle_{b}\right),
$$

when pumped with low pump power $(200 \mathrm{~mW} \mathrm{cw}$ equivalent). $H$ and $V$ denote horizontal and vertical polarization and $a$ and $b$ are the two spatial emission modes. When pumped with higher pump powers $(700 \mathrm{~mW}$ $\mathrm{cw}$ equivalent), noticeable higher-order emission occurs:

$$
|\psi\rangle_{a, b}=\frac{1}{\sqrt{3}}\left(|H H\rangle_{a}|H H\rangle_{b}+|H V\rangle_{a}|H V\rangle_{b}+|V V\rangle_{a}|V V\rangle_{b}\right) .
$$

This state is guided to two PBS cubes. A detection event in the trigger mode $a^{\prime \prime}$ heralds the generation of either the state $|V\rangle_{a^{\prime}}|V\rangle_{b^{\prime}}|H\rangle_{b^{\prime \prime}}$ or $|H H\rangle_{b^{\prime \prime}}$ (see Fig. 9). Only in the first case are the three modes $a^{\prime}, b^{\prime}$, and $b^{\prime \prime}$ occupied with one single photon, whereas in the latter case mode $b^{\prime \prime}$ is occupied with two photons and mode $b^{\prime}$ with vacuum. Postselection on a fourfold coincidence between modes $a^{\prime \prime}$, $a^{\prime}, b^{\prime}$, and $b^{\prime \prime}$ allows for the heralding of the desired input state where only one photon enters each input mode. The half-wave plates in mode $a^{\prime}$ and $b^{\prime}$ are set to $45^{\circ}$ to render them indistinguishable in polarization from the other photons. This heralding scheme holds independently of any transformation for the photons in modes $a^{\prime}, b^{\prime}$, and $b^{\prime \prime}$ as long as it acts on spatial modes, e.g., consisting of beam splitters and phase shifters only.

\section{Analysis of the threefold coincidence data}

Three photons are inserted into input modes 1, 2, and 4 of the interferometric network. The spectral characteristics of these photons are measured using a single-photon spectrometer (Ocean Optics QE6500) and are in good approximation of Gaussian shape. Note that these spectral data differ slightly compared to the characterization measurements (see Appendix A 3 a).

\begin{tabular}{lcc}
\hline & $\lambda_{c}$ & $\Delta \lambda_{\text {FWHM }}$ \\
\hline In1 & $789.35 \mathrm{~nm}$ & $2.85 \mathrm{~nm}$ \\
In2 & $789.52 \mathrm{~nm}$ & $2.79 \mathrm{~nm}$ \\
In4 & $789.41 \mathrm{~nm}$ & $2.72 \mathrm{~nm}$ \\
\hline
\end{tabular}

TABLE III. Data for the coincidence landscapes of Fig. 3. Three photons are injected into input ports 1, 2, and 4 of the interferometric network. They are recorded as a fourfold coincidence measurement between output modes 1, 3, and 4 and the trigger mode, labeled "Out 134," and output modes 1, 3, and 4 and the trigger mode, labeled "Out 345." The theoretical prediction, "theoretical," for the output probability to record a certain event for a pair of temporal delays $\Delta \tau_{1}$ and $\Delta \tau_{2}$ is calculated using Eq. (A16). The experimentally acquired count rates, "experimental," are normalized to unit vectors and fitted to the theoretical data resulting in a goodness of fit of

\begin{tabular}{|c|c|c|c|c|c|c|}
\hline & $\Delta \tau_{1}$ & $\Delta \tau_{2}$ & Theoretical & Experimental & $\chi_{\text {red }}^{2}$ & Count rate \\
\hline \multirow{6}{*}{ Out 134} & $0 \mathrm{fs}$ & $130 \mathrm{fs}$ & $3.41 \%$ & $3.17 \% \pm 0.26 \%$ & \multirow{6}{*}{1.38} & \multirow{6}{*}{$\approx 10 \mathrm{mHz}$} \\
\hline & $0 \mathrm{fs}$ & $-870 \mathrm{fs}$ & $1.89 \%$ & $2.18 \% \pm 0.19 \%$ & & \\
\hline & $-300 \mathrm{fs}$ & $-170 \mathrm{fs}$ & $3.13 \%$ & $2.99 \% \pm 0.25 \%$ & & \\
\hline & $-1000 \mathrm{fs}$ & $-870 \mathrm{fs}$ & $2.95 \%$ & $2.96 \% \pm 0.26 \%$ & & \\
\hline & $-1000 \mathrm{fs}$ & $130 \mathrm{fs}$ & $2.20 \%$ & $2.51 \% \pm 0.21 \%$ & & \\
\hline & $-1000 \mathrm{fs}$ & $1130 \mathrm{fs}$ & $2.73 \%$ & $2.72 \% \pm 0.31 \%$ & & \\
\hline \multirow{6}{*}{ Out 345} & $0 \mathrm{fs}$ & $130 \mathrm{fs}$ & $14.19 \%$ & $14.73 \% \pm 0.93 \%$ & \multirow{6}{*}{1.10} & \multirow{6}{*}{$\approx 80 \mathrm{mHz}$} \\
\hline & $0 \mathrm{fs}$ & $-870 \mathrm{fs}$ & $23.69 \%$ & $24.01 \% \pm 0.84 \%$ & & \\
\hline & $-300 \mathrm{fs}$ & $-170 \mathrm{fs}$ & $17.67 \%$ & $19.10 \% \pm 0.98 \%$ & & \\
\hline & $-1000 \mathrm{fs}$ & $-870 \mathrm{fs}$ & $25.09 \%$ & $24.01 \% \pm 0.85 \%$ & & \\
\hline & $-1000 \mathrm{fs}$ & $130 \mathrm{fs}$ & $21.14 \%$ & $21.32 \% \pm 0.80 \%$ & & \\
\hline & $-1000 \mathrm{fs}$ & $1130 \mathrm{fs}$ & $31.40 \%$ & $30.85 \% \pm 1.40 \%$ & & \\
\hline
\end{tabular}
$\chi_{\text {red }}^{2}=1.38$ and $\chi_{\text {red }}^{2}=1.10$, respectively. The experimental errors are standard deviations over 19 independent measurements. 
TABLE IV. Data for the normalized output probability distributions of Fig. 4. The column "Figure" specifies the temporal delays of each part of Fig. 4, whereas the column " $T_{i j k}$ " refers to the scattering submatrix of Eq. (A12). The experimentally acquired count rates are normalized to unit vectors, then fitted to the theoretical output probability and given under column "Experiment in \%." The errors are standard deviations over 19 independent measurements. The last column, "Theory in \%," shows the theoretical output probability according to Eq. (A16). The contribution of the permanent, the immanant, and the determinant to this theoretical value is given in the columns "per in \%," ... imm in \%," and "det in \%," respectively.

\begin{tabular}{|c|c|c|c|c|c|c|}
\hline Figure & $T_{i j k}$ & Experiment in $\%$ & per in $\%$ & $\mathrm{imm}$ in $\%$ & det in $\%$ & Theory in $\%$ \\
\hline \multirow[t]{4}{*}{ 4(a) } & 245 & $1.46 \pm 0.39$ & 1.72 & 0.13 & 0.00 & 1.86 \\
\hline & 235 & $10.02 \pm 0.83$ & 11.32 & 0.44 & 0.00 & 11.76 \\
\hline & 123 & $46.8 \pm 3.00$ & 33.38 & 11.97 & 0.00 & 45.36 \\
\hline & 345 & $0.47 \pm 0.20$ & 0.03 & 0.16 & 0.00 & 0.19 \\
\hline \multirow[t]{2}{*}{$\Delta \tau_{1}=0 \mathrm{fs}$} & 234 & $7.24 \pm 0.80$ & 7.08 & 0.77 & 0.00 & 7.85 \\
\hline & 134 & $6.69 \pm 0.71$ & 6.30 & 1.54 & 0.00 & 7.85 \\
\hline \multirow[t]{4}{*}{$\Delta \tau_{2}=130 \mathrm{fs}$} & 125 & $7.96 \pm 0.89$ & 5.21 & 2.87 & 0.00 & 8.08 \\
\hline & 145 & $1.69 \pm 0.40$ & 1.41 & 0.13 & 0.00 & 1.55 \\
\hline & 135 & $8.01 \pm 0.77$ & 3.98 & 1.10 & 0.00 & 5.08 \\
\hline & 124 & $9.71 \pm 0.94$ & 8.95 & 1.50 & 0.00 & 10.45 \\
\hline \multirow[t]{4}{*}{ 4(b) } & 245 & $1.35 \pm 0.24$ & 0.93 & 0.59 & 0.01 & 1.53 \\
\hline & 235 & $7.93 \pm 0.68$ & 6.11 & 2.45 & 0.11 & 8.67 \\
\hline & 123 & $50.86 \pm 2.60$ & 18.02 & 29.62 & 1.19 & 48.82 \\
\hline & 345 & $0.78 \pm 0.16$ & 0.02 & 0.64 & 0.01 & 0.66 \\
\hline \multirow[t]{2}{*}{$\Delta \tau_{1}=-300 \mathrm{fs}$} & 234 & $6.00 \pm 0.41$ & 3.82 & 3.04 & 0.03 & 6.89 \\
\hline & 134 & $5.12 \pm 0.58$ & 3.40 & 1.21 & 0.01 & 4.61 \\
\hline \multirow[t]{4}{*}{$\Delta \tau_{2}=-170 \mathrm{fs}$} & 125 & $8.07 \pm 0.74$ & 2.81 & 3.69 & 0.03 & 6.53 \\
\hline & 145 & $1.86 \pm 0.26$ & 0.76 & 1.20 & 0.02 & 1.98 \\
\hline & 135 & $8.64 \pm 0.68$ & 2.15 & 8.86 & 0.14 & 11.15 \\
\hline & 124 & $9.39 \pm 0.59$ & 4.83 & 4.16 & 0.16 & 9.15 \\
\hline \multirow[t]{4}{*}{ 4(c) } & 245 & $1.17 \pm 0.26$ & 0.37 & 0.77 & 0.05 & 1.19 \\
\hline & 235 & $6.59 \pm 0.58$ & 2.46 & 3.35 & 0.59 & 6.40 \\
\hline & 123 & $53.64 \pm 1.90$ & 7.26 & 40.72 & 6.54 & 54.52 \\
\hline & 345 & $0.92 \pm 0.20$ & 0.01 & 0.96 & 0.03 & 1.00 \\
\hline \multirow[t]{2}{*}{$\Delta \tau_{1}=-1000 \mathrm{fs}$} & 234 & $5.29 \pm 0.43$ & 1.54 & 3.63 & 0.15 & 5.32 \\
\hline & 134 & $4.06 \pm 0.40$ & 1.37 & 2.43 & 0.04 & 3.84 \\
\hline \multirow[t]{4}{*}{$\Delta \tau_{2}=-870 \mathrm{fs}$} & 125 & $8.19 \pm 0.64$ & 1.13 & 6.26 & 0.19 & 7.58 \\
\hline & 145 & $1.57 \pm 0.22$ & 0.31 & 1.24 & 0.12 & 1.67 \\
\hline & 135 & $9.91 \pm 0.74$ & 0.87 & 8.03 & 0.78 & 9.68 \\
\hline & 124 & $8.67 \pm 1.00$ & 1.95 & 5.97 & 0.89 & 8.80 \\
\hline \multirow[t]{4}{*}{$4(d)$} & 245 & $0.92 \pm 0.23$ & 0.19 & 0.51 & 0.17 & 0.86 \\
\hline & 235 & $5.12 \pm 0.58$ & 1.21 & 1.91 & 1.97 & 5.09 \\
\hline & 123 & $58.26 \pm 2.70$ & 3.58 & 33.36 & 21.69 & 58.63 \\
\hline & 345 & $0.60 \pm 0.09$ & 0.00 & 0.56 & 0.11 & 0.68 \\
\hline \multirow[t]{2}{*}{$\Delta \tau_{1}=-1000 \mathrm{fs}$} & 234 & $4.17 \pm 0.39$ & 0.76 & 2.85 & 0.50 & 4.11 \\
\hline & 134 & $4.03 \pm 0.51$ & 0.68 & 2.79 & 0.12 & 3.59 \\
\hline \multirow[t]{4}{*}{$\Delta \tau_{2}=1130 \mathrm{fs}$} & 125 & $7.38 \pm 0.62$ & 0.56 & 6.00 & 0.64 & 7.19 \\
\hline & 145 & $1.32 \pm 0.28$ & 0.15 & 0.91 & 0.39 & 1.45 \\
\hline & 135 & $10.00 \pm 0.38$ & 0.43 & 7.15 & 2.57 & 10.15 \\
\hline & 124 & $8.18 \pm 0.71$ & 0.96 & 4.33 & 2.95 & 8.25 \\
\hline
\end{tabular}

These spectral data allow us to express the mode-overlap integrals as a function of the time delays $\Delta \tau_{1}$ and $\Delta \tau_{2}$ between the first and second photon and the second and third photon, respectively. The theoretical prediction for the output probability in any of the ten threefold output ports is then calculated using Eq. (A15). Consequently, each $3 \times 3$ submatrix $R$ is constituted by matrix elements selected by the input and output ports. The output probability [see Eq. (A15)] of any landscape contains a constant term and four terms proportional to different mode-overlap functions. By sampling six points of pairwise temporal delay of $\Delta \tau_{1}$ and $\Delta \tau_{2}, P 1-P 6$ in the table below, contributions of each of these terms can be assessed. These six points are shown here. 


\begin{tabular}{lrr}
\hline & \multicolumn{1}{c}{$\Delta \tau_{1}$} & \multicolumn{1}{c}{$\Delta \tau_{2}$} \\
\hline$P 1$ & $0 \mathrm{fs}$ & $130 \mathrm{fs}$ \\
$P 2$ & $0 \mathrm{fs}$ & $-870 \mathrm{fs}$ \\
$P 3$ & $-300 \mathrm{fs}$ & $-170 \mathrm{fs}$ \\
$P 4$ & $-1000 \mathrm{fs}$ & $-870 \mathrm{fs}$ \\
$P 5$ & $-1000 \mathrm{fs}$ & $130 \mathrm{fs}$ \\
$P 6$ & $-1000 \mathrm{fs}$ & $1130 \mathrm{fs}$ \\
\hline
\end{tabular}

An offset of $\Delta \tau_{\text {off }}=130$ fs is introduced in the temporal delay mode $\Delta \tau_{2}$; other than that the delays are set to combinations of $0,-300$, and \pm 1000 fs. The precision of the temporal alignment is estimated to be $\pm 16 \mathrm{fs}$. In one measurement run the points $P 1-P 6$ are recorded consecutively for $2 \mathrm{~h}$ each. To account for effects of drift, this order is reversed in the next measurement run; therefore, the points are recorded in the order $P 6-P 1$. The fourfold count rates range from 1 to $100 \mathrm{mHz}$, dependent on the output combination. In between each measurement run the setup is realigned to optimize for maximal count rates. In order to obtain sufficient statistics, the whole data acquisition is repeated over 19 measurement runs for a total of $228 \mathrm{~h}$.

As Poissonian error modeling results in too optimistic error bars in the case of long data acquisition due to multiple sources of error, we adapt the error modeling. The 19 measurements are independent runs; therefore, their mean and standard deviation of the mean provide more useful information. Each individual measurement run is represented as a six-dimensional vector, with the $i$ th entry of the vector containing the fourfold counts of the $P_{i}$ th delay point integrated over $2 \mathrm{~h}$. These vectors can then be normalized to unit vectors thereby obtaining relative output probabilities. The mean and the standard deviation of the mean can now be calculated for each of the six delay points. Ultimately, the overlap with the theoretical prediction is obtained by a least-squared minimization weighted with the standard deviations. Here, a linear scaling factor is introduced relating the relative experimental probabilities to the absolute theoretical ones (see Table III). The goodness of fit is calculated using the reduced $\chi^{2}$. The number of degrees of freedom in this case is $\nu=6-2=4$.

The experimental data for the four different scenarios of BosonSampling affected by distinguishability, shown in Fig. 4, are recorded using the same method as above. Table IV shows the experimental data and theoretical prediction for Figs. 4(a)-4(d).

[1] J. L. O'Brien, A. Furusawa, and J. Vučković, Photonic Quantum Technologies, Nat. Photonics 3, 687 (2009).

[2] M. C. Tichy, H.-T. Lim, Y.-S. Ra, F. Mintert, Y.-H. Kim, and A. Buchleitner, Four-Photon Indistinguishability Transition, Phys. Rev. A 83, 062111 (2011).

[3] Y.-S. Ra, M. C. Tichy, H.-T. Lim, O. Kwon, F. Mintert, A. Buchleitner, and Y.-H. Kim, Nonmonotonic
Quantum-to-Classical Transition in Multiparticle Interference, Proc. Natl. Acad. Sci. U.S.A. 110, 1227 (2013).

[4] M. C. Tichy, Sampling of Partially Distinguishable Bosons and the Relation to the Multidimensional Permanent, Phys. Rev. A 91, 022316 (2015).

[5] V.S. Shchesnovich, Partial Indistinguishability Theory for Multiphoton Experiments in Multiport Devices, Phys. Rev. A 91, 013844 (2015).

[6] V. Tamma and S. Laibacher, Multiboson Correlation Interferometry with Arbitrary Single-Photon Pure States, Phys. Rev. Lett. 114, 243601 (2015).

[7] H. de Guise, S.-H. Tan, I. P. Poulin, and B. C. Sanders, Coincidence Landscapes for Three-Channel Linear Optical Networks, Phys. Rev. A 89, 063819 (2014).

[8] S. Aaronson and A. Arkhipov, The Computational Complexity of Linear Optics, in Proceedings of the 43rd Annual ACM Symposium on Theory of Computing (Association for Computing Machinery, New York, 2011), pp. 333-342.

[9] P. Bürgisser, The Computational Complexity of Immanants, SIAM J. Comput. 30, 1023 (2000).

[10] C. K. Hong, Z. Y. Ou, and L. Mandel, Measurement of Subpicosecond Time Intervals between Two Photons by Interference, Phys. Rev. Lett. 59, 2044 (1987).

[11] S.-H. Tan, Y. Y. Gao, H. de Guise, and B. C. Sanders, $S U(3)$ Quantum Interferometry with Single-Photon Input Pulses, Phys. Rev. Lett. 110, 113603 (2013).

[12] D. E. Littlewood and A. R. Richardson, Group Characters and Algebra, Phil. Trans. R. Soc. A 233, 99 (1934).

[13] J.-Q. Chen, H. Pang, and F. Wang, Group Representation Theory for Physicists, 2nd ed. (World Scientific, Singapore, 2002).

[14] M. A. Nielsen and I. L. Chuang, Quantum Computation and Quantum Information (Cambridge University Press, Cambridge, England, 2010).

[15] A. E. Lita, A. J. Miller, and S. W. Nam, Counting NearInfrared Single-Photons with 95\% Efficiency, Opt. Express 16, 3032 (2008).

[16] Z. Zhou, S. Jahanmirinejad, F. Mattioli, D. Sahin, G. Frucci, A. Gaggero, R. Leoni, and A. Fiore, Superconducting Series Nanowire Detector Counting Up to Twelve Photons, Opt. Express 22, 3475 (2014).

[17] F. Marsili et al., Detecting Single Infrared Photons with 93\% System Efficiency, Nat. Photonics 7, 210 (2013).

[18] A. Leverrier and R. García-Patrón, Analysis of Circuit Imperfections in Boson Sampling, Quantum Inf. Comput. 15, 0489 (2015).

[19] P. P. Rohde and T.C. Ralph, Error Tolerance of the Boson-Sampling Model for Linear Optics Quantum Computing, Phys. Rev. A 85, 022332 (2012).

[20] P. P. Rohde, Optical Quantum Computing with Photons of Arbitrarily Low Fidelity and Purity, Phys. Rev. A 86, 052321 (2012).

[21] P. P. Rohde, K. R. Motes, P. A. Knott, and W. J. Munro, Will Boson-Sampling Ever Disprove the Extended ChurchTuring Thesis?, arXiv:1401.2199.

[22] Peter Bürgisser, The Computational Complexity of Immanants, SIAM J. Comput. 30, 1023 (2000).

[23] J.-L. Brylinski and R. Brylinski, Complexity and Completeness of Immanants, arXiv:cs/0301024. 
[24] E. Knill, R. Laflamme, and G. J. Milburn, A Scheme for Efficient Quantum Computation with Linear Optics, Nature (London) 409, 46 (2001).

[25] T. B. Pittman, B. C. Jacobs, and J. D. Franson, Probabilistic Quantum Logic Operations Using Polarizing Beam Splitters, Phys. Rev. A 64, 062311 (2001).

[26] B. J. Metcalf et al., Quantum Teleportation on a Photonic Chip, Nat. Photonics 8, 770 (2014).

[27] J. Carolan et al., Universal Linear Optics, Science 349, 711 (2015).

[28] P. G. Kwiat, K. Mattle, H. Weinfurter, A. Zeilinger, A. V. Sergienko, and Y. Shih, New High-Intensity Source of Polarization-Entangled Photon Pairs, Phys. Rev. Lett. 75, 4337 (1995).

[29] K. Itoh, W. Watanabe, S. Nolte, and C. B. Schaffer, Ultrafast Processes for Bulk Modification of Transparent Materials, MRS Bull. 31, 620 (2006).

[30] G. D. Marshall, A. Politi, J. C. F. Matthews, P. Dekker, M. Ams, M. J. Withford, and J. L. O'Brien, Laser Written
Waveguide Photonic Quantum Circuits, Opt. Express 17, 12546 (2009).

[31] H. Fearn and R. Loudon, Theory of Two-Photon Interference, J. Opt. Soc. Am. B 6, 917 (1989).

[32] M. Reck, A. Zeilinger, H. J. Bernstein, and P. Bertani, Experimental Realization of Any Discrete Unitary Operator, Phys. Rev. Lett. 73, 58 (1994).

[33] M. Tillmann, B. Dakić, R. Heilmann, S. Nolte, A. Szameit, and P. Walther, Experimental Boson Sampling, Nat. Photonics 7, 540 (2013).

[34] A. Laing and J. L. O'Brien, Super-Stable Tomography of Any Linear Optical Device, arXiv:1208.2868.

[35] J. Taylor, Introduction to Error Analysis, the Study of Uncertainties in Physical Measurements (University Science Books, New York, 1997).

[36] S. Rahimi-Keshari, M. A. Broome, R. Fickler, A. Fedrizzi, T. C. Ralph, and A. G. White, Direct Characterization of Linear-Optical Networks, Opt. Express 21, 13450 (2013). 